ARTICLE

Received 12 Nov 2013 | Accepted 27 May 2014 | Published 24 Jun 2014

DOI: $10.1038 /$ ncomms5229

\title{
p38 MAPK-inhibited dendritic cells induce superior antitumour immune responses and overcome regulatory T-cell-mediated immunosuppression
}

Yong Lu ${ }^{1}$, Mingjun Zhang ${ }^{1}$, Siqing Wang ${ }^{2}$, Bangxing Hong ${ }^{1}$, Zhiqiang Wang ${ }^{3}$, Haiyan $\mathrm{Li}^{1}$, Yuhuan Zheng ${ }^{1}$, Jing Yang ${ }^{3}$, Richard E. Davis ${ }^{3}$, Jianfei Qian ${ }^{1}$, Jian $\mathrm{Hou}^{4} \&$ Qing Yi ${ }^{1}$

Dendritic cell (DC)-based cancer immunotherapy is a promising method, but so far has demonstrated limited clinical benefits. Regulatory T cells (Tregs) represent a major obstacle to cancer immunotherapy approaches. Here we show that inhibiting p38 MAPK during DC differentiation enables DCs to activate tumour-specific effector T cells (Teff), inhibiting the conversion of Treg and compromising Treg inhibitory effects on Teff. Inhibition of p38 MAPK in DCs lowers expression of PPAR $\gamma$, activating p50 and upregulating OX40L expression in DCs. OX4OL/OX40 interactions between DCs and Teff and/or Treg are critical for priming effective and therapeutic antitumour responses. Similarly, p38 MAPK inhibition also augments the T-cell stimulatory capacity of human monocyte-derived DCs in the presence of Treg. These findings contribute to ongoing efforts to improve DC-based immunotherapy in human cancers.

\footnotetext{
${ }^{1}$ Department of Cancer Biology, Lerner Research Institute, Cleveland Clinic, Cleveland, Ohio 44195, USA. ${ }^{2}$ Institute of Translational Medicine, The First Hospital, Jilin University, Changchun 130061, China. ${ }^{3}$ Department of Lymphoma/Myeloma, Division of Cancer Medicine, The University of Texas MD Anderson Cancer Center, Houston, Texas 77030, USA. ${ }^{4}$ Chang Zheng Hospital, The Second Military University, Shanghai 200438, China. Correspondence and requests for materials should be addressed to J.H. (email: houjian@medmail.com.cn) or to Q.Y. (email: yiq@ccf.org)
} 
$\mathrm{D}$ endritic cell (DC)-based immunotherapy holds great promise for treating malignancies ${ }^{1}$. However, the results of most DC-based clinical trials have been disappointing ${ }^{2}$. In cancer patients, $\mathrm{CD} 4{ }^{+} \mathrm{CD} 25^{+}$Foxp $3{ }^{+}$regulatory $\mathrm{T}$ cells (Tregs) accumulate in tumours and secondary lymphoid organs by the mechanisms of expansion of Treg or the conversion of effector $\mathrm{T}$ cells $(\mathrm{Teff})^{3,4}$. Tumour-associated Tregs likely contribute to the suppressive milieu, leading to the suppression of T-cell responses partly via the inhibition of DC functions ${ }^{5}$. Thus, modulating the suppressive function of Treg is essential for induction of effective antitumour immunity 6,7 .

Among the possible strategies to target Treg in vivo, depletion of $\mathrm{CD} 25^{+} \mathrm{T}$ cells by anti-CD25 antibodies may not be a viable approach because both Treg and activated Teff are depleted, inducing conversion of peripheral precursors into Treg ${ }^{4,8-10}$. A unique surface marker for Treg has not been identified, and an alternative approach to target Treg is the use of cytotoxic $\mathrm{T}$ lymphocyte antigen 4 (CTLA4)-blocking antibodies or glucocorticoid-induced tumour necrosis factor receptor (GITR) receptor agonist antibodies to revert Treg-mediated suppress$\operatorname{ion}^{8,11}$. However, these strategies can result in over-activating of nonspecific T-cell immunity and have been linked to severe autoimmunity in multiple organs, thus limiting their further clinical applications ${ }^{12,13}$. Therefore, a more desirable approach is needed to modulate the suppressive function of Treg and activate Teff in an antigen-specific manner.

In an earlier study, we demonstrated that lipopolysaccharide (LPS)-induced activation of p38 mitogen-activated protein kinase (MAPK) has a detrimental effect on the generation of immature DCs (iDCs) in vitro ${ }^{14}$. We and others have also shown that tumour-derived suppressive factors inhibit the differentiation and function of DCs by upregulating p38 MAPK activity in DC precursors in both murine tumour models and cancer patients ${ }^{15-}$ 18. These abnormalities in the phenotype and T-cell stimulatory capacity of DCs could be restored by inhibiting p38 MAPK activity in progenitor cells ${ }^{15-18}$. Based on these observations, we hypothesized that p38 MAPK may attenuate antigen presentation and have an essential role in maintenance of self-tolerance in DC.

In this study, we inhibited p38 MAPK activity in DC precursors using the inhibitor SB202190, and inhibitor SB203580 and/or p38 $\alpha$-specific small intefering RNA (siRNA) transfection for confirmation. The inhibition of p38 MAPK results in sharply decreased PPAR $\gamma$ expression in DCs, which reduces functional inhibition of p50 transcriptional activities by PPAR $\gamma$. In turn, p50 activation upregulates surface expression of OX40L on DCs, increasing their immunostimulatory potency, activating antigen-specific Teff, inhibiting Treg conversion and function, and facilitating tumour rejection.

\section{Results}

p38 MAPK-inhibited DCs activate Teff in the presence of Treg. Among the four different $\mathrm{p} 38$ isoenzymes, $\mathrm{p} 38 \alpha$ was the only p38 MAPK isoenzyme detected in the isolated bone marrow (BM) cells and generated iDCs and mature DCs (mDCs) from wildtype C57BL/6 mice (WT-B6 mice) (Supplementary Fig. 1a). We chose to use SB202190 and SB203580, two specific inhibitors of the $\alpha$ - and $\beta$-isoforms of p38 MAPK ${ }^{19}$. Treatment with $1.5 \mu \mathrm{M}$ SB202190 or SB203580, but not the inactive analogue SB202474 (ref. 20), was sufficient to inhibit the p38 MAPK activity in BM cells, as determined by our previous studies ${ }^{16}$, and by the blockage of the phosphorylation of its downstream kinase MAPKAPK-2 (Supplementary Fig. 1b). To examine the role of p38 MAPK in regulating DC generation and maturation (all generated from BM cells from WT-B6 mice unless indicated specifically), flow cytometry was used to analyse the surface expression of various molecules related to antigen presentation. SB202190- and SB203580-treated, but not SB202474-treated, iDCs expressed higher levels of DC-related molecules compared with control iDCs (Supplementary Fig. 1c). After maturation, SB202190-treated mDCs (mSBDCs) and SB203580-treated mDCs (mSB80DCs) showed significantly higher levels of major histocompatibility complex (MHC) class II, CD80, CD86, CD40 and OX40L than dimethylsulphoxide (DMSO)-treated control mDCs and SB202474-treated mDCs (mSB74DCs) (Fig. 1a,b and Supplementary Fig. 1d). In addition, p38 MAPK inhibition enhanced interleukin (IL)-12 production by mSBDCs and mSB80DCs (Supplementary Fig. 1e). All these cells produced undetectable or very low levels of cytokines such as IL-6, TNF- $\alpha$, IL-10 and interferon (IFN)- $\gamma$ (ranging from 0 to $50 \mathrm{pg} \mathrm{ml}^{-1}$ ). In line with these results, knockdown of p38 $\alpha$ in DC progenitor cells by $\mathrm{p} 38 \alpha$-specific siRNA during the differentiation also induced the upregulated expression of DC-related surface molecules as compared with nonspecific siRNA-treated (Si-non-) mDCs (Supplementary Fig. 2). Collectively, these results suggested that p38 MAPK negatively regulated the generation and immunogenicity of BM-derived DCs (BMDCs).

The enhanced immunogenicity of mSBDCs and mSB80DCs may enable them to promote proliferation of activate Teff in the presence of Treg. We used a carboxyfluorescein diacetate succinimidyl ester (CFSE) dilution assay to test the proliferation of Teff. As shown in Fig. 1c and Supplementary Fig. 3, mSBDCs, mSB80DCs or p38 $\alpha$-specific siRNA-treated (Si-p38 $\alpha$-) mDCs induced a profound proliferation of $\mathrm{CD} 4{ }^{+}$Teff in the presence of Treg, whereas the proliferation of $\mathrm{CD} 4{ }^{+}$Teff stimulated by mDCs, mSB74DCs or Si-non-mDCs was largely inhibited. Notably, the proliferation of $\mathrm{CD} 4{ }^{+}$Teff stimulated by mSBDCs or mSB80DCs in the presence of Treg was even stronger than those stimulated by mDCs in the absence of Treg. We found that $\mathrm{CD} 4{ }^{+}$Teff proliferation induced by mSBDCs, mSB80DCs or Si-p38 $\alpha$-mDCs was inhibited significantly by Treg when OX40Lblocking monoclonal antibody ( $\mathrm{mAb}$ ) was added (Fig. 1d and Supplementary Fig. 3). We also screened other molecules that might have contributed to this effect by using functional grade blocking mAbs targeting CD80, CD86, CD40 or IL-12 (Supplementary Fig. 1f-h), and excluded their involvement (Fig. 1d). These data suggest that the enhanced stimulatory function of mSBDCs and mSB80DCs is mediated exclusively by upregulated OX40L expression.

Flow cytometry analysis showed that mSBDCs expressed significantly higher levels of surface OX40L, and Teff stimulated by mSBDCs expressed significantly upregulated OX40L than T cells stimulated by mDCs (Supplementary Fig. 4a,b). As Tregs do not express $\mathrm{OX}_{40 \mathrm{~L}^{22}}$, we next examined the contribution of cell expression of OX40L. We used OX40L-specific siRNA-treated mSBDCs (Si-OX40L-mSBDCs; Supplementary Fig. 4b), and found that $\mathrm{CD}^{+}{ }^{+}$Teff proliferation induced by Si-OX40LmSBDCs was subject to Treg-mediated suppression (Fig. 1e). This Si-OX40L-mSBDC-induced Teff proliferation decreased further to the level of $\mathrm{mDC}$-induced $\mathrm{CD} 4{ }^{+}$Teff proliferation in the presence of Treg when OX40L-blocking antibody was added (Supplementary Fig. 4c), indicating that mSBDC-expressed OX40L had a major role in counteracting Treg-mediated suppression of Teff proliferation, and that Teff-expressed OX40L may also be important. These results suggested that mSBDCs may be able to induce tumour-specific T-cell responses even in the presence of Treg.

OX40 costimulation inhibits Treg and activates Teff. In the absence of Treg, the OX40/OX40L signalling appears to only have a minimal effect on proliferation of $\mathrm{CD} 4{ }^{+}$Teff (Fig. 2a and 
a
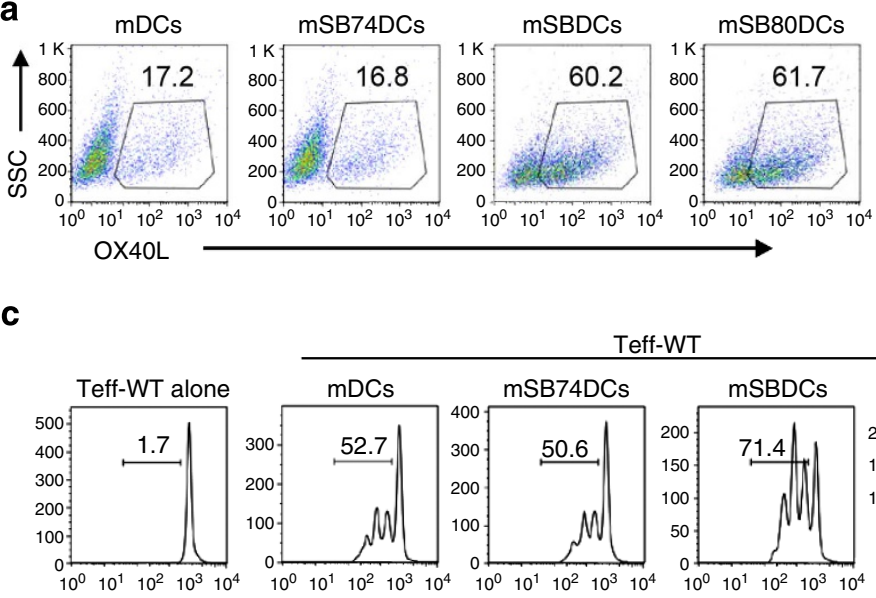

Teff-WT
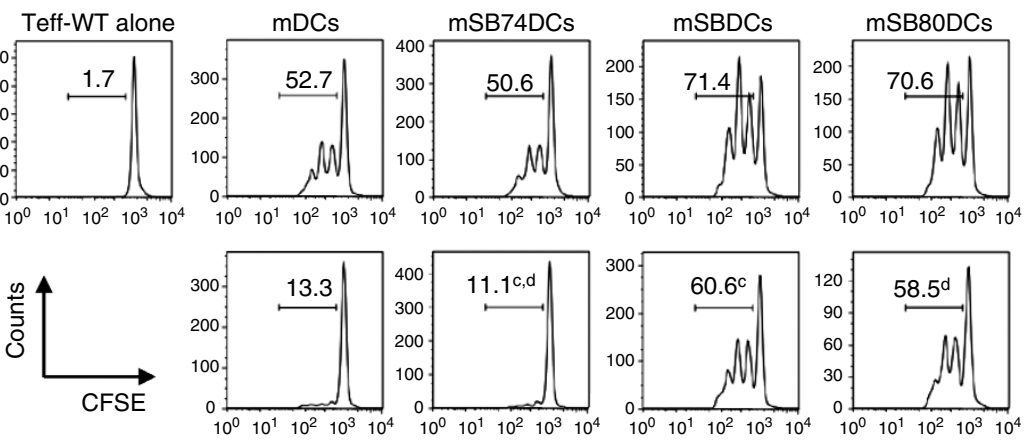

$10^{0} 10^{1} 10^{2} 10^{3} 10^{4}$

$P^{c}=0.0010$

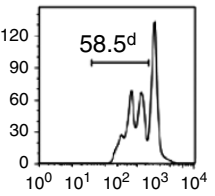

$P^{\mathrm{d}}=0.00086$

+ Treg b

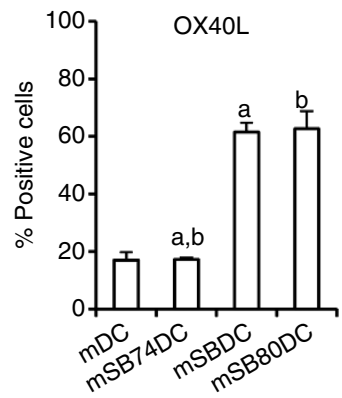

$P \mathrm{a}=2.9 \mathrm{E}-06$

$P^{\mathrm{b}}=0.00021$

d

Teff-WT+Treg
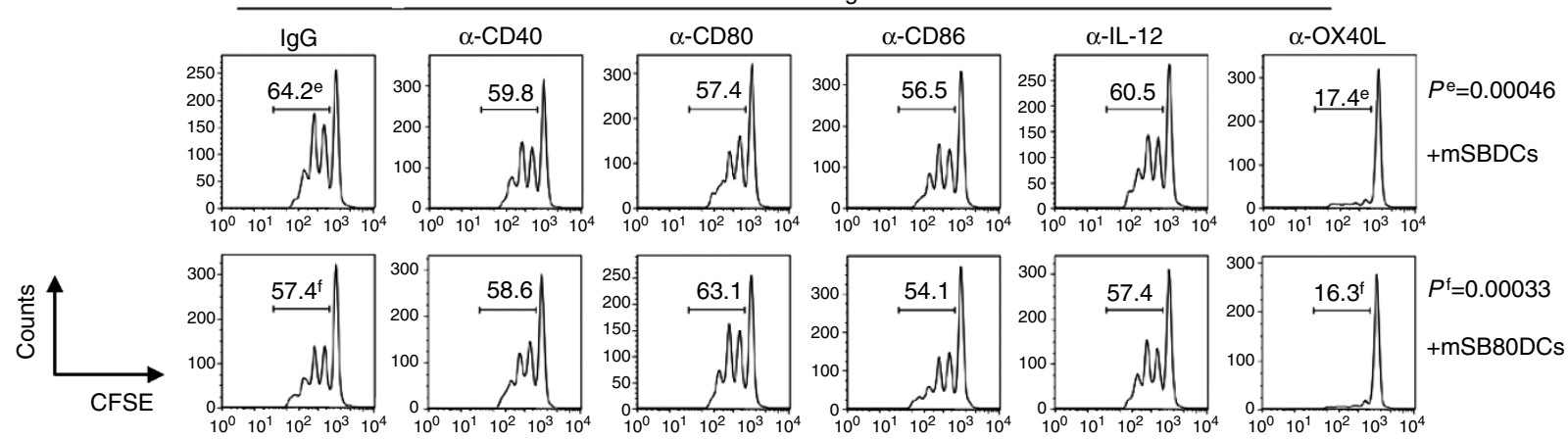

e
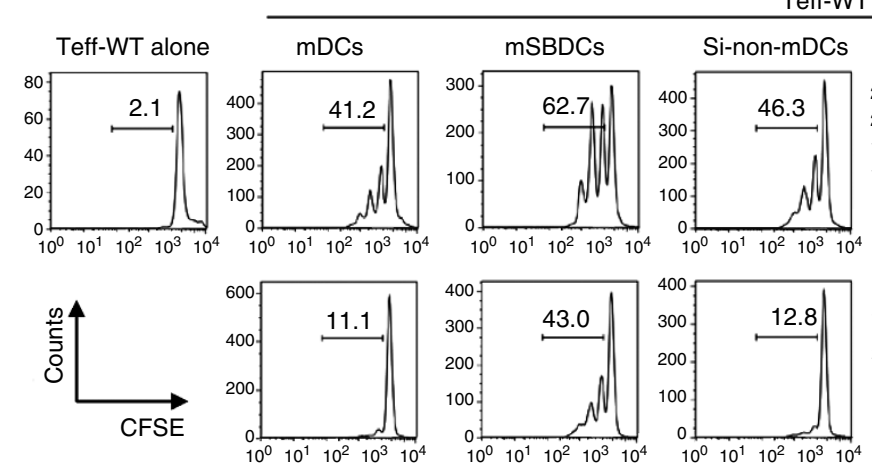

Teff-WT
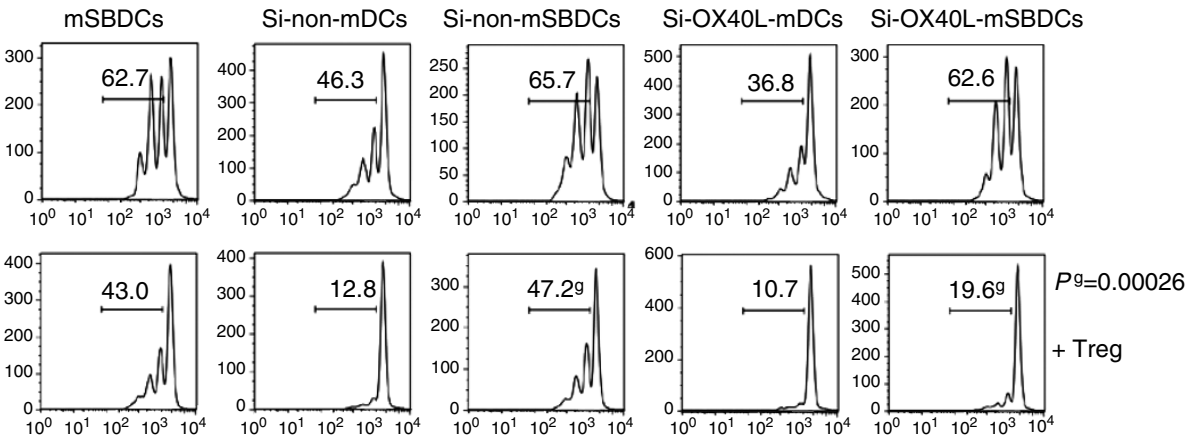

Figure 1 | OX40L expression by $\mathbf{m S B D C}$ is critical for $\mathbf{m S B D C}$-induced Teff proliferation in the presence of Treg. (a) Expression of OX4OL on treated murine BMDCs. Gates were preset on CD11c ${ }^{+}$cells as determined by FACS analysis. Numbers are percentages of OX4OL-expressing DCs. (b) Pooled data $(n=3)$ depicting the percentages of OX40L-positive cells among CD11c ${ }^{+}$mDCs, mSB74DCs, mSBDCs and mSB80DCs. Error bars represent s.d. (c-e) Proliferation of CFSE-labelled wild-type (WT) CD4 ${ }^{+}$CD25 ${ }^{-}$Teff $\left(1 \times 10^{5}\right)$ in the absence or presence of unlabelled CD4 ${ }^{+}$CD25 $5^{+}$Treg $\left(1 \times 10^{5}\right)$ induced by various $\mathrm{mDCs}\left(1 \times 10^{4}\right)$ plus anti-CD3 antibody $\left(1 \mu \mathrm{g} \mathrm{ml} \mathrm{l}^{-1}\right)$. Both Teff and Treg were isolated from spleens of naive C57BL/6 mice.

(c) $\mathrm{CD} 4{ }^{+} \mathrm{CD} 25^{-}$Teff were cocultured with $\mathrm{mDCs}$, mSB74DCs, mSBDCs or mSB80DCs. CD4 ${ }^{+} \mathrm{CD} 25^{+}$Tregs were added as indicated. Sixty hours later, CFSE intensity of Teff was measured by FACS. Numbers indicate percent CFSElo/proliferated Teff. (d) Control lgG or the specific mAbs were added to the coculture of Teff, Treg and mSBDCs or mSB80DCs. Sixty hours later, Teff proliferation was measured by FACS. (e) CD4 ${ }^{+}$CD25 ${ }^{-}$Teff were cocultured with untreated, nonspecific siRNA- (Si-non-) or OX4OL-specific siRNA-treated mDCs or mSBDCs. CD4 ${ }^{+} \mathrm{CD} 25^{+}$Tregs were added as indicated. Sixty hours later, Teff proliferation was measured by FACS. Representative results from one of three performed experiments are shown. $P$ values were calculated with Student's t-test. 
a

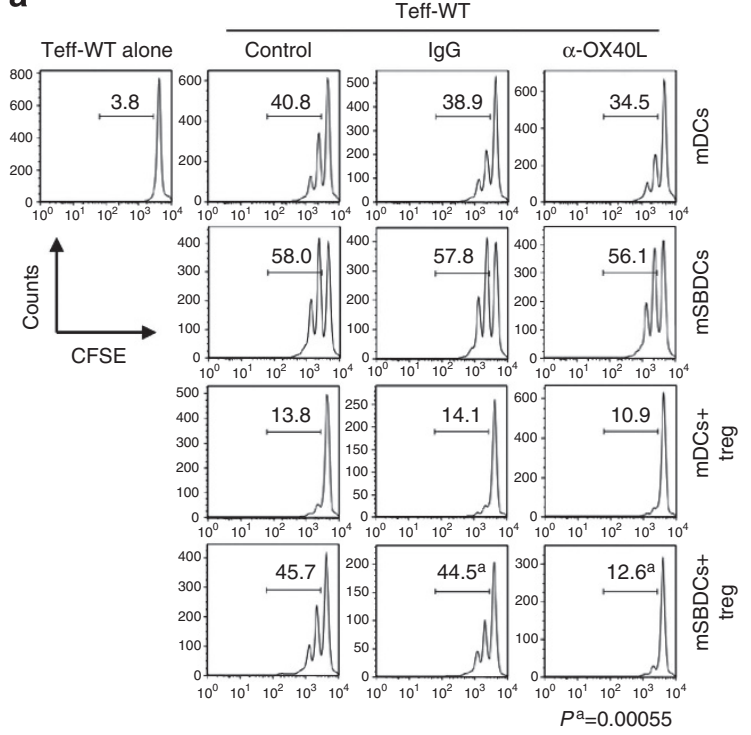

b

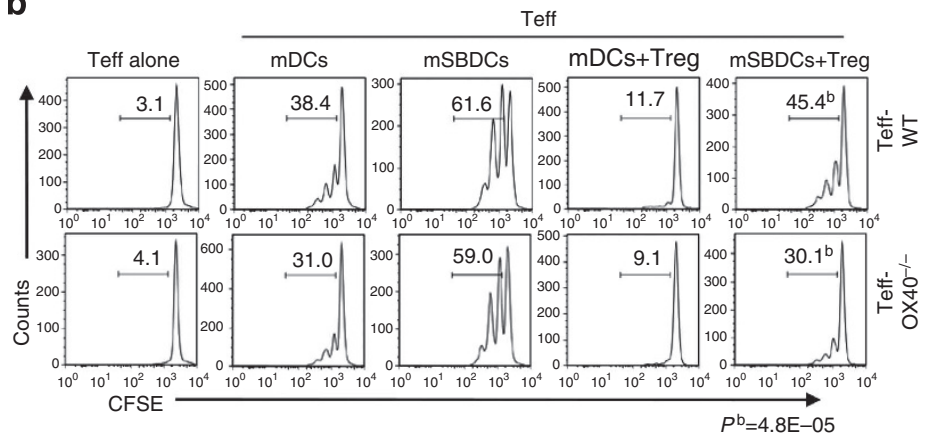

d

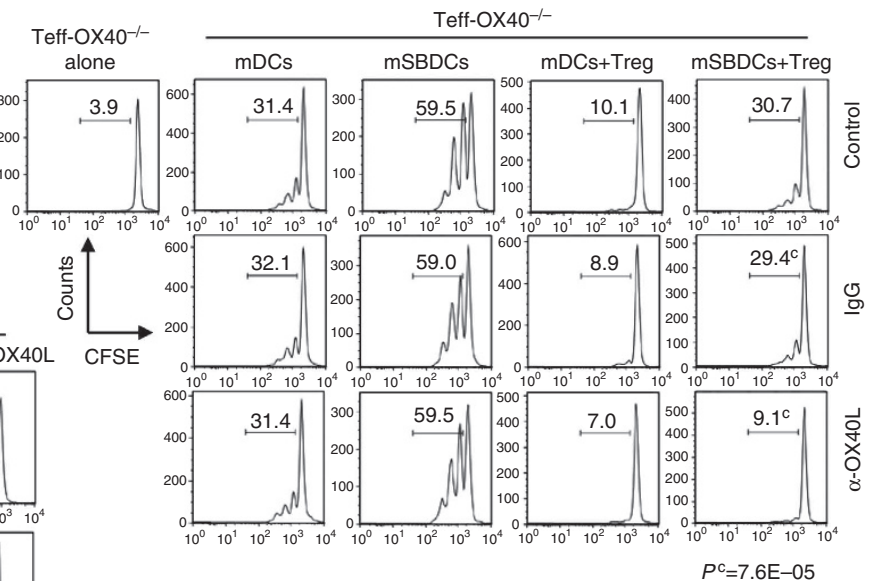

Treg:Teff=1:1 (2:1, bottom row)

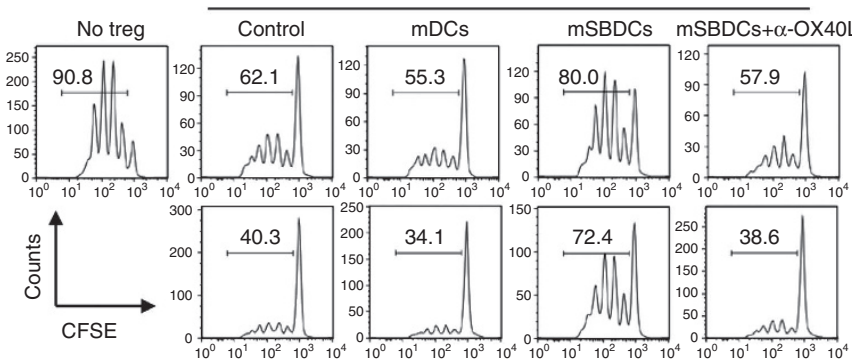

$P^{\mathrm{c}}=7.6 \mathrm{E}-05$

Figure 2 | Role of OX40 signalling in counteracting Treg-mediated suppression by mSBDCs. (a-c) Proliferation of splenic CD4 ${ }^{+} \mathrm{CD} 25^{-}$Teff in the absence or presence of $\mathrm{CD} 4^{+} \mathrm{CD} 25^{+}$Treg isolated from naive C57BL/6 mice. CD4 ${ }^{+} \mathrm{CD} 25^{-}$Teff $\left(1 \times 10^{5}\right)$ from WT C57BL/6 mice (a), WT or OX40-/C57BL/6 mice (b), or OX40-1- C57BL/6 mice (c) were labelled with CFSE and cocultured with mDCs or mSBDCs $\left(1 \times 10^{4}\right)$ plus anti-CD3 antibody $\left(1 \mu \mathrm{g} \mathrm{ml}^{-1}\right)$. Control medium, antibodies and/or unlabelled CD4 ${ }^{+} \mathrm{CD} 25^{+}$Treg $\left(1 \times 10^{5}\right)$ were added as indicated. Sixty hours later, CFSE intensity of Teff was measured by FACS. Numbers indicate percent CFSE $\%$ proliferated Teff. Results are representative of three independent experiments. (d) $\mathrm{CD}_{4}{ }^{+} \mathrm{CD} 25^{+}$Treg isolated from C57BL/6 mice were pretreated with medium, mDCs, mSBDCs or mSBDCs plus anti-OX40L antibody for $5 \mathrm{~h}$. CFSE-labelled OT-II Teff $\left(1 \times 10^{5}\right)$ and peptide-pulsed mDCs $\left(1 \times 10^{4}\right)$ were cocultured in the presence of medium or with $\mathrm{CD}^{+}{ }^{+} \mathrm{CD} 25^{+}$Treg isolated from the pretreatment condition at the indicated Treg to OT-II Teff ratios. Seventy-two hours later, CFSE intensity of OT-II Teff was examined by FACS. Representative data from one of two repeated experiments are shown. $P$ values were calculated with Student's $t$-test.

Supplementary Fig. 3). As Tregs constitutively express OX40 and activated Teff but not DCs express OX40 (refs 21,23-25), we next determined the contribution of cell expression of OX40 for the activation of Teff by mSBDCs in the presence of Treg. We first examined the role of OX40 expression on Teff. As shown in Fig. $2 \mathrm{~b}$, there was only a marginal decrease in the proliferation of OX40-1- CD4 ${ }^{+}$Teff, as compared with WT Teff, in response to mSBDCs or mDCs. However, when Tregs were present, the proliferation of the $\mathrm{OX}_{40}{ }^{-1-} \mathrm{CD} 4{ }^{+}$Teff stimulated by mSBDCs decreased significantly as compared with those of WT Teff, indicating that loss of OX40 on CD4 ${ }^{+}$Teff rendered them more sensitive to Treg-mediated suppression as compared with their WT counterparts when stimulated by mSBDCs. In addition, we also investigated the role of Treg-expressed OX40 in Tregmediated suppressive function. Since $\mathrm{OX} 40^{-1-}$ Treg from $\mathrm{OX}_{40} 0^{-1-}$ mice showed impaired suppressive function ${ }^{24}$, we could not use $O X 40^{-1-}$ Treg in our studies. We cocultured mSBDCs, OX40 $40^{-1-} \mathrm{CD} 4^{+}$Teff and Treg, in which OX40/ OX40L costimulation only occurs between mSBDCs and Treg, and found that the proliferation of $\mathrm{OX}_{40} 0^{-1-} \mathrm{CD} 4^{+}$Teff induced by mSBDCs was significantly inhibited when OX40Lblocking antibody was added (Fig. 2c), suggesting that interaction between mSBDCs and Treg via OX40/OX40L costimulation may lead to reduced inhibitory ability of Treg. Furthermore, mSBDCpretreated Treg became less effective in inhibiting the proliferation of OT-II Teff compared with control or mDCpretreated Treg in an OX40L-dependent manner (Fig. 2d). Similar results were also obtained from CD8 ${ }^{+} \mathrm{T}$ cells stimulated by mSBDCs in the presence or absence of Treg (Supplementary Fig. 5). Thus, these findings indicate the importance of OX40/ OX40L signalling in mSBDC-induced resistance to Treg inhibition of Teff proliferation.

OX40 costimulation by mSBDCs inhibits Teff conversion to Treg. We found a significantly smaller Treg population $\left(\mathrm{CD} 4^{+}\right.$ $\mathrm{CD} 25^{+}$Foxp $^{+}$) among CD4 ${ }^{+} \mathrm{T}$ cells in the lymph nodes of mSBDC-immunized mice as compared with $\mathrm{mDC}$-immunized mice (Fig. 3a), indicating that mSBDCs inhibited the expansion of preexisting Treg and/or conversion of $\mathrm{CD} 4{ }^{+} \mathrm{CD} 25^{-}$Teff to Treg. Since mSBDCs and mDCs had similar stimulatory effects on expansion of Treg (Supplementary Fig. 6a), we hypothesized that mSBDCs may inhibit the conversion of $\mathrm{CD} 4^{+} \mathrm{CD} 25^{-}$Teff to Treg. As shown in Fig. 3b, mSBDCs generated significantly 
a

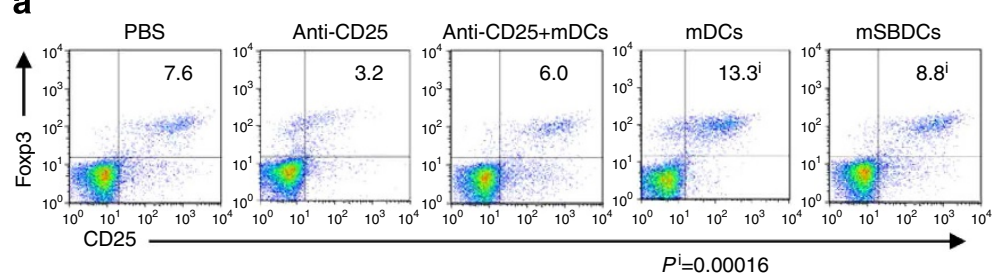

d

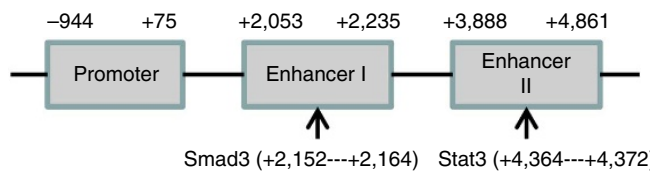

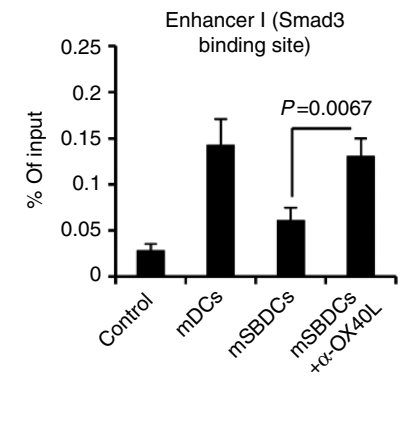

e f

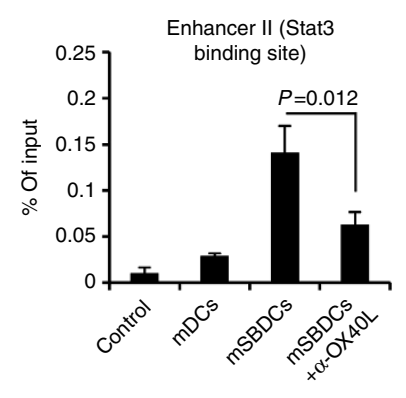

b

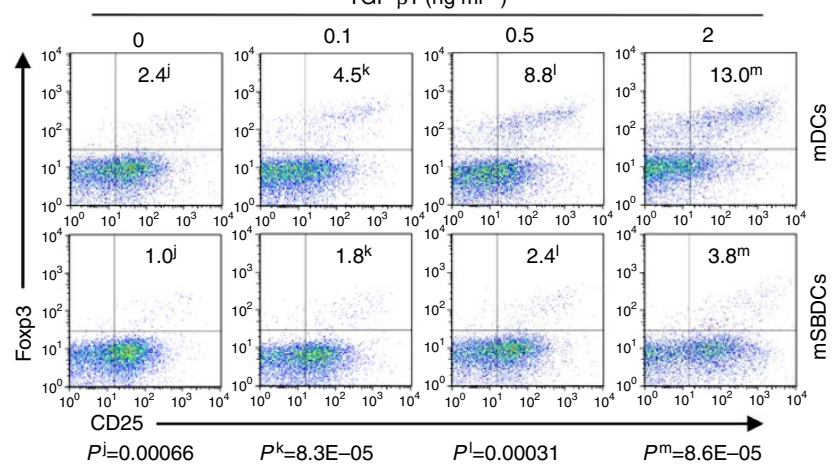

C

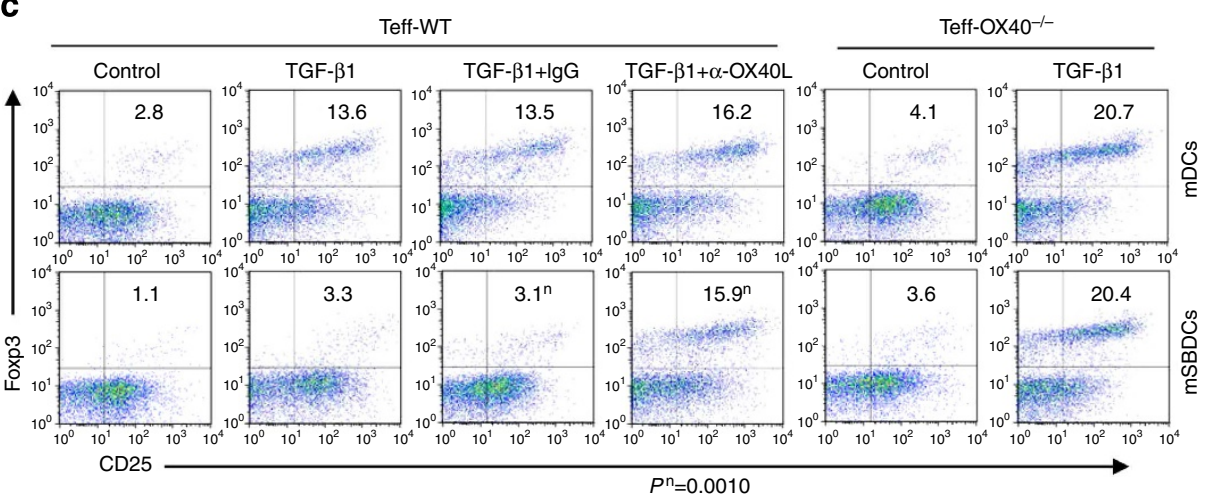

Figure 3 | mSBDCs inhibit Teff to Treg conversion by $\mathbf{0 X 4 0}$ costimulation. (a) FACS analysis of Treg population in pooled draining lymph nodes 10 days after the second immunization. C57BL/6 mice were immunized twice with $1 \times 10^{6}$ OT-II peptide-loaded mDCs or mSBDCs with or without anti-CD25 antibody treatment. Numbers represent the percentages of $\mathrm{CD} 25^{+}$Foxp3 ${ }^{+}$Treg among gated $\mathrm{CD} 4{ }^{+} \mathrm{T}$ cells. (b,c) Conversion of Teff into Treg was suppressed by mSBDC stimulation. CD4 ${ }^{+} \mathrm{CD} 25^{-}$Foxp3 $3^{-}$Teff $\left(1 \times 10^{5}\right)$ isolated from WT or OX40-/- C57BL/6 mice were cocultured with mDCs or mSBDCs $\left(1 \times 10^{4}\right)$ in the presence of anti-CD3 antibody $\left(1 \mu \mathrm{gl}^{-1}\right)$ and IL-2 $\left(20 \mathrm{U} \mathrm{ml}^{-1}\right)$ with the addition of varying doses of TGF- $\beta 1$ (b) or TGF- $\beta 1$

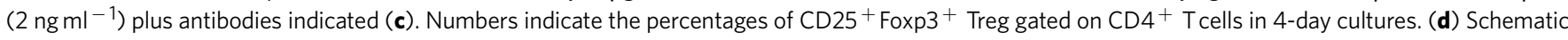
diagram of Smad3 binding site in Foxp3 enhancer I region and Stat3 binding site in Foxp3 enhancer II region. (e,f) $C D 4{ }^{+} \mathrm{CD} 25^{-}$Teff were cocultured with mDCs or mSBDCs in the presence of TGF- $\beta 1$ and IL-2 for 4 days. CD4 ${ }^{+}$T cells were purified for analyses. (e) ChIP assay was performed using Smad3 antibody or control IgG. Precipitated DNA was subjected to RT-PCR using primers targeting enhancer I region. Error bars represent s.d. $(n=3)$. (f) ChIP assay was performed using Stat3 antibody or control lgG. Precipitated DNA was subjected to RT-PCR using primers targeting enhancer II region. Error bars represent s.d. $(n=3)$. Data are representative of three $(\mathbf{a}-\mathbf{c})$ or two $(\mathbf{e}, \mathbf{f})$ independent experiments. $P$ values were calculated with $S$ tudent's $t$-test.

fewer $\mathrm{CD} 4{ }^{+} \mathrm{CD} 25^{+}$Foxp3 ${ }^{+}$Treg than mDCs in vitro cultures of $\mathrm{CD} 4^{+} \mathrm{CD} 25^{-}$Teff without exogenous TGF- $\beta 1$. Surprisingly, when TGF- $\beta 1$ induced a large number of Treg from Teff when stimulated with mDCs, mSB74DCs or Si-non-mDCs, mSBDCs, mSB80DCs and Si-p38 $\alpha$-mDCs strongly inhibited the conversion of Teff to Treg (Fig. 3b and Supplementary Fig. 6b,c).

To shed light on the mechanisms driving these effects, we screened IFN- $\gamma$, IL-4, IL-12, IL-6, OX40L and other molecules in mSBDCs for their potential contribution to the inhibition of Teff conversion to Treg ${ }^{7,26}$. Our results showed that $\mathrm{mAbs}$ blocking OX40L, but not other molecules (Supplementary Fig. 6d), almost completely abrogated the inhibitory effect of mSBDCs on Treg conversion (Fig. 3c), indicating that OX40/OX40L signalling may be involved. In addition, mSBDCs and mDCs had similar ability in conversion of $O X 40^{-1-}$ Teff into Treg in the presence or absence of exogenous TGF- $\beta 1$ (Fig. 3c). Moreover, in vivo blocking OX40/OX40L pathway by injecting OX40L-blocking antibodies restored the generation of Treg population in tumourdraining lymph nodes from mSBDC-immunized mice to the same level of mice immunized with mDCs (Supplementary Fig. 7). To elucidate the mechanism underlying mSBDC-inhibited conversion of Treg, we investigated the binding of phosphorylated (p)Smad3 to the enhancer region I of Foxp3 promoter, in light of a previous study showing that the binding of $\mathrm{pSmad} 3$ to the enhancer region I increases Foxp3 transcription, whereas the binding of pStat 3 to the conserved enhancer region II works as a gene silencer during Foxp3 transcription (Fig. 3d) ${ }^{27}$. Our results show that, although the level of pSmad3 and pStat 3 did not 
change in $\mathrm{CD}^{+}{ }^{+} \mathrm{T}$ cells cocultured with mDCs or mSBDCs in the presence of TGF- $\beta 1$ (Supplementary Fig. 6e), binding of pSmad 3 to the enhancer region I and pStat 3 to the enhancer region II were significantly suppressed or enhanced, respectively, in mSBDC-cocultured $\mathrm{CD}^{+}{ }^{+} \mathrm{T}$ cells in an OX40L-dependent manner (Fig. 3e,f). These results suggest epigenetic inhibition of Treg generation by mSBDCs. Together, these findings show that OX40/OX40L signalling provided by mSBDCs may contribute to the reduced generation of immunosuppressive cells in vitro and in vivo.

Sustained p50 activation mediates OX40L expression in mSBDCs. Our results indicate that both enhanced stimulatory function of mSBDCs and the inhibition of Teff conversion into Treg by mSBDCs are mediated exclusively by OX40L. We next elucidated the molecular mechanism underlying p38 inhibitionmediated upregulation of OX40L in mSBDCs. Because nuclear factor- $\kappa \mathrm{B}(\mathrm{NF}-\kappa \mathrm{B})$ pathway contributes to the immunogenicity of DCs and $O x 40 l$ promoter contains a potential NF- $\kappa B$ binding site (5'-GGGAAATTCA-3' ${ }^{\prime},-84$ to -75 , upstream of $\left.O x 40 l\right)$, we hypothesized that the NF- $\kappa \mathrm{B}$ pathway has an important role in the increased expression of OX40L on mSBDCs. By comparing $\mathrm{NF}-\kappa \mathrm{B}$ molecules in the nuclear fractions of mDCs and mSBDCs, we found that inhibition of p38 MAPK resulted in slightly increased nuclear translocation of RelA and RelB and robust nuclear translocation of p50 (Fig. 4a and Supplementary Fig. 8). Similarly, as compared with untreated mDCs and Si-non-mDCs, Si-p38 $\alpha$-mDCs also demonstrated a substantially upregulated nuclear translocation of p50 (Supplementary Fig. 9a). Next, by chromatin immunoprecipitation (ChIP) assay, we detected an increased physiological binding of RelA, RelB and especially p50 to the Ox40l promoter in mSBDCs (Fig. 4b). To examine whether these NF- $\kappa \mathrm{B}$ molecules could activate the $\mathrm{O} x 40 \mathrm{l}$ promoter, we performed luciferase reporter gene assays in HEK 293T cells. RelA, RelB or p52 alone did not activate the Ox40l promoter, whereas p50 together with RelB, and to a lesser extent, with RelA or c-Rel, did (Fig. 4c). Considering that these NF- $\kappa B$ molecules were increased in the nuclear fraction of mSBDCs, these data indicate that nuclear translocation of $\mathrm{p} 50$, which formed transcriptionally active complexes with RelA, c-Rel and especially with RelB, was responsible for the upregulation of OX40L expression in $\mathrm{mSBDCs}$.

To further verify the p50-dependent upregulation of surface expression of OX40L on mSBDCs, we generated BMDCs from BM cells of $p 50^{-1-}$ B6-mice. The expression of OX40L on $p 50^{-/-}$iDCs and $p 50^{-/-}$mDCs was significantly reduced as compared with those generated from BM cells of WT-B6 mice (Fig. 4d,e). Furthermore, SB202190-treated $p 50^{-/-}$iDCs or $p 50^{-1-}$ mDCs failed to upregulate the expression of surface OX40L. The surface OX40L expression level was similar between $p 50^{-1-} \mathrm{mDCs}$ and $p 50^{-/-}$mSBDCs, confirming a crucial role of p50 in modulating OX40L expression of mSBDCs.

Inhibition of PPAR $\gamma$ contributes to p50 activation in mSBDCs. Next, we examined the signalling pathways that may lead to

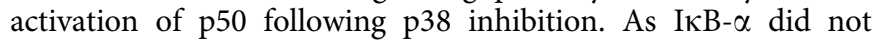

a

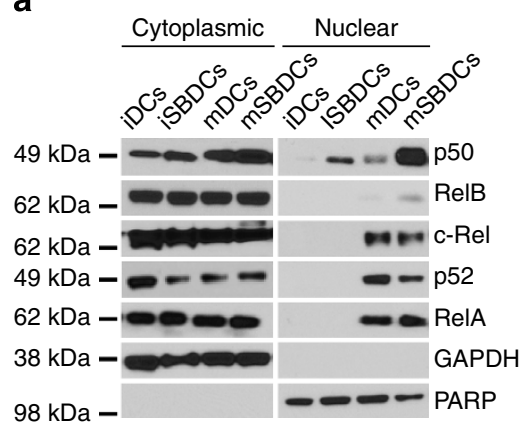

b

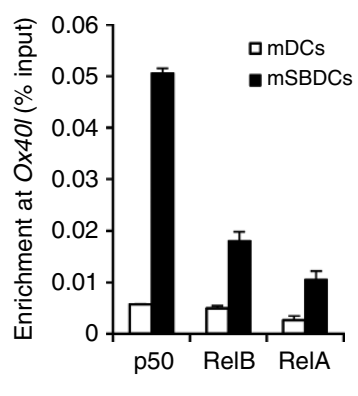

C

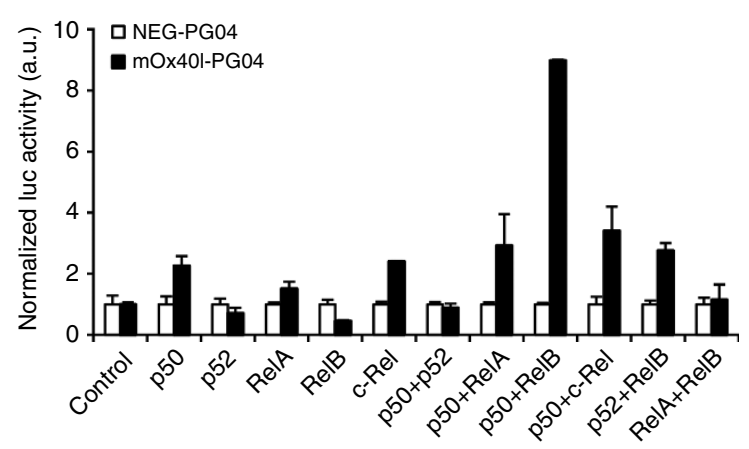

e d

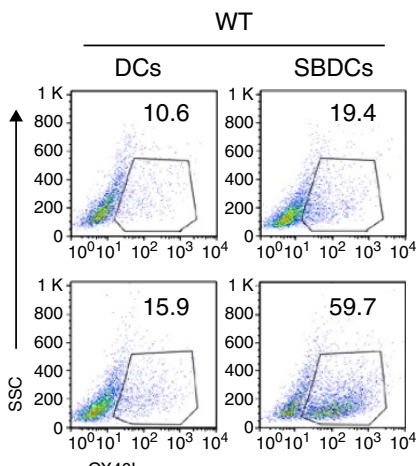

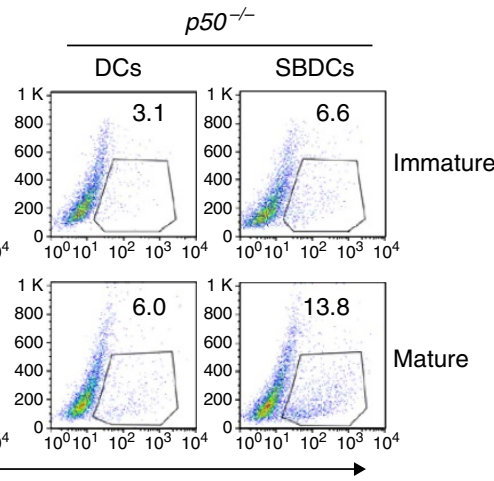

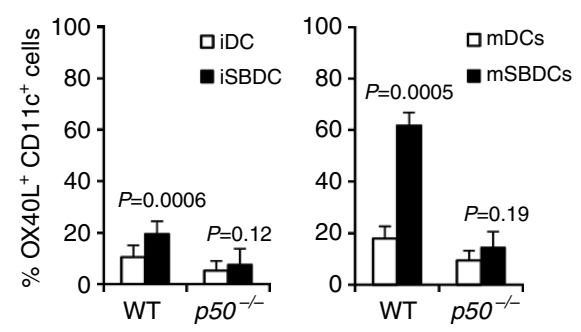

Figure 4 | Inhibition of p38 MAPK activates p50-dependent pathways that result in increased surface expression of OX40L on mSBDCs. (a) Western blot analysis of the cytoplasmic and nuclear localization of NF-KB molecules in iDCs, mDCs, iSBDCs and mSBDCs. (b) ChIP assay of p50, RelA and RelB binding to the $\mathrm{O} \times 40 \mathrm{O}$ promoter in $\mathrm{mDCs}$ and $\mathrm{mSBDCs}$. Error bars represent s.d. $(n=3)$. (c) Luciferase reporter assay showed NF- $\mathrm{kB}-\mathrm{dependent}$ activation of the Ox40/ promoter in HEK 293T cells. HEK 293T cells in triplicated wells were transiently transfected with mouse Ox40I promoter-inserted vector $\mathrm{mOx} 4 \mathrm{OI}-\mathrm{PGO} 4$ or empty plasmid NEG-PG04, followed by transfection with vectors for NF-kB molecules. Error bars represent s.d. $(n=3)$.

(d) Surface expression of OX4OL on iDCs, iSBDCs, mDCs and mSBDCs generated from WT and p50-/- C57BL/6 mice analysed by FACS. Numbers

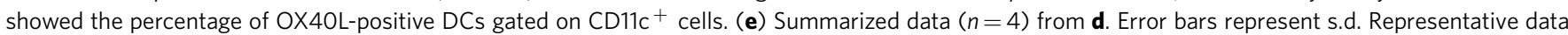
from one of two repeated experiments are shown. $P$ values were calculated with Student's $t$-test. 
decrease in mSBDCs (Supplementary Fig. 9b), we performed gene array analysis to investigate the potential molecules that may have contributed to the nuclear translocation of p50. We hypothesized that the decreased PPAR $\gamma$ expression we observed in gene array analysis may have a role in activating p50 (ref. 22). First, we verified that the protein level of PPAR $\gamma$ was sharply decreased in mSBDCs as compared with mDCs (Fig. $5 \mathrm{a}$ and Supplementary Fig. 10) and in Si-p38 $\alpha$-mDCs as compared with Si-non-mDCs (Supplementary Fig. 9c). Also, the level of CCAAT/enhancerbinding protein $(\mathrm{pC} / \mathrm{EBP} \beta)$, a known downstream target of $\mathrm{p} 38$ MAPK $^{28}$, was reduced in mSBDCs and in Si-p38 $\alpha$-mDCs (Fig. 5a and Supplementary Figs $9 \mathrm{c}$ and 10 ), which could potentially be responsible for decreased levels of PPAR $\gamma$, considering it is the master transcriptional factor of PPAR $\gamma^{29}$. As a confirmation for the observations, knockdown of C/EBP $\beta$ by siRNA diminished PPAR $\gamma$ level in mDCs (Fig. 5b and Supplementary Fig. 10).

Second, we measured PPAR $\gamma$ activity by detection of mRNA levels of $A p 2$, which has been described as a direct PPAR $\gamma$ $\operatorname{target}^{30}$. As expected, inhibition of p38 MAPK reduced Ap2 levels in mDCs as well as the PPAR $\gamma$ inhibitor, whereas both rosiglitazone (RGZ), a PPAR $\gamma$ activator, and the p38 activator, anisomycin $^{31}$, enhanced $A p 2$ expression (Fig. 5c). In addition, inhibition of p38 MAPK failed to suppress PPAR $\gamma$ activity directly induced by RGZ (Fig. 5c). By analysis of the nuclear translocation of $\mathrm{p} 50$, we found that $\mathrm{p} 50$ activity was retroactively correlated with PPAR $\gamma$ activity in mDCs (Fig. 5c,d and Supplementary Fig. 10). Moreover, we detected the presence of p50 in PPAR $\gamma$ immunoprecipitate from $\mathrm{mDCs}$ but not from mSBDCs (Fig. 5e and Supplementary Fig. 10), suggesting a possible mechanism of transrepression of p50 activity by PPAR $\gamma$ by complex formation ${ }^{22}$, which antagonizes p50 signalling in DCs.

Finally, we also found that PPAR $\gamma$ inhibitor-induced p50 activation led to upregulated expression of surface OX40L on mDCs, while p50 deficiency abrogated this effect (Fig. 5f,g). As inhibition of p38 MAPK could not reverse RGZ-mediated PPAR $\gamma$ activation, RGZ-treated mSBDCs failed to express the high levels of OX40L observed on mSBDCs (Fig. 5f,g). These results suggest that inhibition of p38 MAPK suppresses PPAR $\gamma$ expression and activity in mSBDCs by decreasing C/EBP $\beta$ phosphorylation, a

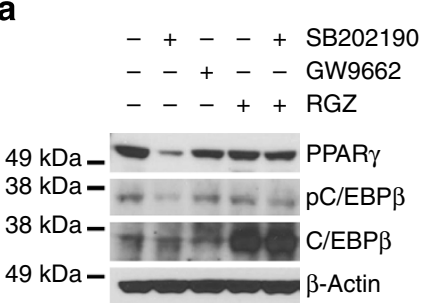

b

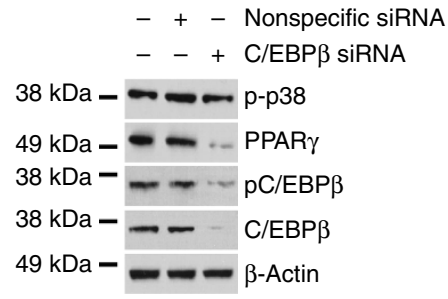

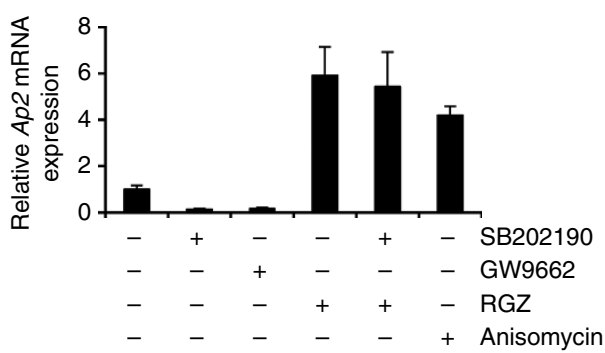

d

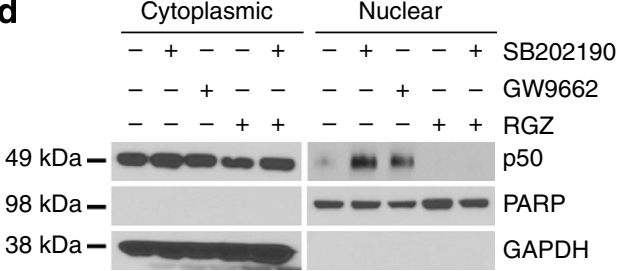

e

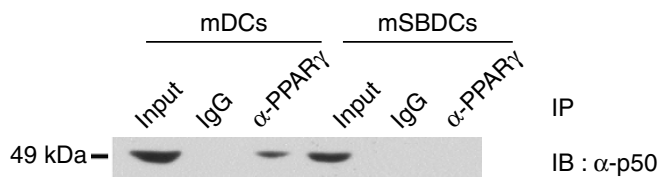

f

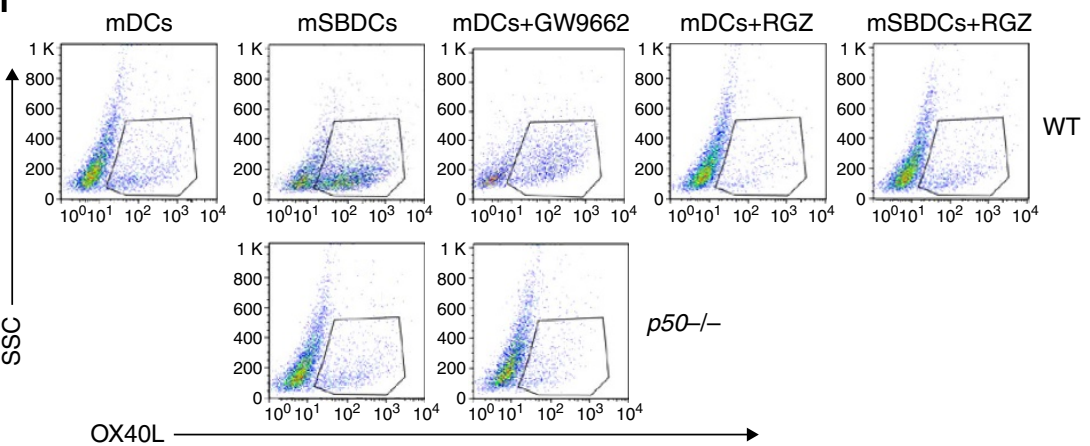

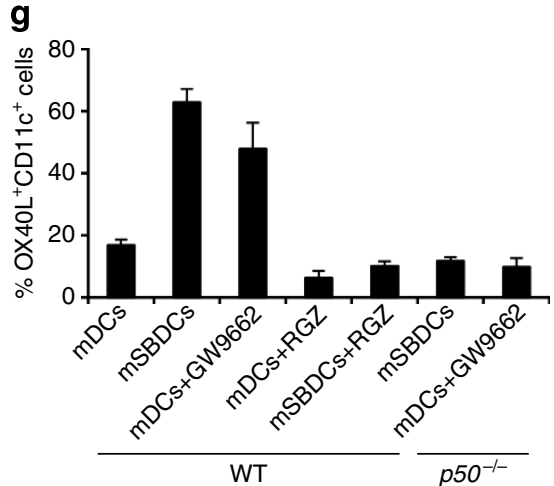

Figure 5 | Downregulation of PPAR $\gamma$ expression/activity in mSBDCs contributes to the p50-mediated increased expression of surface OX40L. (a) Western blot analysis of the PPAR $\gamma, \mathrm{pC} / \mathrm{EBP} \beta$ and C/EBP $\beta$ in $\mathrm{mDCs}$ and $\mathrm{mSBDC}$ s treated with or without p38 inhibitor SB202190, PPAR $\gamma$ inhibitor GW9662 and/or PPAR $\gamma$ activator RGZ. (b) The protein level of PPAR $\gamma, \mathrm{pC} / \mathrm{EBP} \beta$ and C/EBP $\beta$ in mDCs treated with C/EBP $\beta$-specific siRNA. (c) RT-PCR analysis of Ap2 mRNA expression in mDCs treated with or without p38 inhibitor SB202190, PPAR $\gamma$ inhibitor GW9662, PPAR $\gamma$ activator RGZ, and/or p38 activator anisomycin. Data shown $(n=3)$ were normalized to the Gapdh gene. Error bars represent s.d. (d) Western blot analysis of the cytoplasmic and nuclear localization of p50 molecule in treated mDCs and mSBDCs. (e) Coimmunoprecipitation of endogenous p50 and PPAR 7 . mDCs and mSBDCs lysates were immunoprecipitated with PPAR $\gamma$ antibody or isotype control lgG and immunoblotted with p50 antibody. (f) Surface expression of OX4OL on treated mDCs and mSBDCs generated from WT and $p 50^{-/-}$mice analysed by FACS. Numbers showed the percentage of OX4OL-positive DCs gated on $\mathrm{CD} 11 \mathrm{c}^{+}$cells. (g) Summarized data $(n=4)$ from $\mathbf{f}$. Error bars represent s.d. Representative data from one of two performed experiments are shown. $P$ values were calculated with Student's $t$-test. 
thereby leading to reduced functional inhibition of PPAR $\gamma$ on p50 transcription factor activity and increased expression of OX40L on mSBDCs.

mSBDCs overcome Treg-mediated immunosuppression in vivo. The observed regulatory role of p38 MAPK in DC differentiation and immunogenicity promoted us to examine whether p38 inhibited-mDC can induce potent antigen-specific antitumour immunity and facilitate tumour rejection. Similar to other reports of the B16-OVA ${ }^{32}$ and B16 (ref. 33) tumour model, mice were immunized with OT-I peptide-pulsed mSBDCs (mSBDCs-OT-I), mDCs-OT-I, mSBDCs-OT-II, mDCs-OT-II, mSBDC-TRP2 or mDC-TRP2 at days 3 and 10 after tumour cell inoculation. Groups of mice were depleted of CD25 ${ }^{+}$Treg with an intraperitoneal injection of CD25-specific antibody or treated with combined Treg cell depletion (3 days before immunization) and DC immunization. We (Supplementary Fig. 11a) and others ${ }^{34}$ have found that a single injection of CD25-specific antibody can deplete $>90 \%$ of Treg for a week by examining $\mathrm{CD}_{2}{ }^{+}$Foxp3 $^{+} \mathrm{T}$ cells in the spleens. Tumour growth was significantly inhibited by immunization with mSBDCs-OT-I, mSBDCs-OT-II or mSBDC-TRP2, whereas immunization with non-pulsed mSBDCs or Treg depletion had marginal effects, and mDCs-OT-I, mDCs-OT-II or mDC-TRP2 immunization had moderate antitumour effects (Fig. 6a-c and Supplementary Fig. 11b). Notably, mSBDCs immunization alone was at least as effective as the combined treatments of Treg depletion and $\mathrm{mDC}$ immunization in inhibiting the growth of pre-established tumour (Fig. 6a-c). In addition, the enhanced antitumour effects of antigen-pulsed mSBDCs were correlated with the significantly higher frequencies of antigen-specific $\mathrm{CD}^{+}{ }^{+}$or $\mathrm{CD}^{+}$IFN- $\gamma$-secreting $\mathrm{T}$ cells in spleens and the significantly increased CTL activity in immunized mice (Fig. $6 \mathrm{~d}-\mathrm{f}$ and Supplementary Fig. 11c). Importantly, Si-p38 $\alpha$-mDCs-OT-I, mSBDCs-OT-I and mSB80DCs-OT-I, but not the Si-non-mDCsOT-I, mDCs-OT-I and mSB74DCs-OT-I, could prevent the outgrowth of pre-established B16-OVA tumour (Supplementary Fig. $11 \mathrm{~d}-\mathrm{g}$ ), further demonstrating the crucial role of inhibiting p38 MAPK signalling in DCs to induce superior antitumour response after immunization.

We also examined the contribution of OX40L signal delivered by $\mathrm{mSBDCs}$ in activating T-cell immunity. We found that OX40L antibody treatment could abrogate the proliferation of activated OT-II $\mathrm{T}$ cells induced by mSBDC-OT-II in tumour-draining lymph nodes (Supplementary Fig. 12), and substantially reduced the ability of mSBDCs-OT-II and mSBDCs-OT-I to induce tumour regression (Supplementary Fig. 13). Furthermore, $p 50^{-1-}$ mSBDCs-OT-I expressed low levels of OX40L and failed to induce an effective antitumour response compared with mSBDCs-OT-I from WT mice (Supplementary Fig. 11f). These observations reveal the importance of OX40 costimulation initiated by $\mathrm{mSBDCs}$ in inducing antitumour immunity in vivo.

mSBDCs activate antitumour immunity in the effector phase. We then examined the in vivo effects of mSBDCs in the effector phase of antitumour immune response. Tumour-infiltrating lymphocytes (TILs) were isolated from B16-OVA tumour-bearing C57BL/6 mice receiving different treatments. The numbers of tumour-infiltrating, IFN- $\gamma$-secreting $\mathrm{CD}^{+}$or $\mathrm{CD}^{+} \mathrm{T}$ cells were significantly increased in mSBDCs-OT-I- or mSBDCs-OTII-immunized mice, respectively, than the other groups (Fig. 7a). We observed a significantly upregulated expression of OX40 and OX40L on both $\mathrm{CD}^{+}{ }^{+}$and CD4 ${ }^{+}$Teff that might amplify the OX40/OX40L signal on the effector phase of antitumour immune response (Fig. 7b). Tumour-infiltrating $\mathrm{CD} 8{ }^{+}$or $\mathrm{CD} 4{ }^{+} \mathrm{CD} 25^{-}$
(Fig. 7c) Teff isolated from mice immunized with mSBDCs-OT-I or mSBDCs-OT-II, respectively, had significantly enhanced proliferation when restimulated in vitro with OT-I or OT-II peptideloaded mDCs than those from other groups, even in the presence of $\mathrm{CD} 4{ }^{+} \mathrm{CD} 25^{+}$Treg from naive mice. We also analysed tumour-infiltrating $\mathrm{CD} 4{ }^{+} \mathrm{CD} 25^{+}$Foxp $3^{+}$Treg population, and to our surprise, mSBDCs-OT-I or mSBDCs-OT-II immunization resulted in significantly decreased percentages of Treg in gated $\mathrm{CD}^{+}$TILs (Fig. 7d). Moreover, CD $4^{+} \mathrm{CD} 25^{\text {hi }}$ Treg isolated from TILs in mSBDCs-OT-II-immunized mice had significantly decreased suppressive function as compared with those from mDC-immunized or phosphate buffered saline (PBS)-treated mice in inhibiting the proliferation of $\mathrm{CD} 4{ }^{+} \mathrm{CD} 25^{-}$OT-II cells stimulated with mDCs-OVA (Fig. 7e). In addition, as compared with mSBDCs-OT-I, mSB74DCs-OT-I and $p 50^{-1-}$ mSBDCsOT-I displayed significantly decreased capacity to activate tumour-infiltrating Teff, reduce Treg cell population and compromise the suppressive function of tumour-infiltrating Treg (Supplementary Fig. 14). Taken together, these data indicate that the inhibited p38 MAPK signalling, as well as the subsequently activated p50 NF- $\mathrm{B}$ pathway, are indispensable for mSBDCstimulated activation of tumour-infiltrating Teff during the effector phase.

The role of p38 MAPK in human monocyte-derived DCs. We further asked whether p38 MAPK inhibition could enhance the Teff-stimulatory capacity of human monocyte-derived DCs (hMoDCs) in the presence of Treg. Inhibition of p38 MAPK during the differentiation of h-MoDCs by $1 \mu \mathrm{M}$ SB202190 or SB203580, but not SB202474 or DMSO, significantly upregulated the surface expression of OX40L on these cells after maturation (Fig. 8a). In the absence of Treg, the OX40/OX40L signalling appears to only have a minimal effect on the proliferation of alloreactive CD4 ${ }^{+}$ Teff induced by p38 MAPK inhibitor-treated h-mMoDCs (Fig. 8b). Importantly, similar to murine p38 MAPK-inhibited BMDCs, p38 MAPK inhibitor-treated h-mMoDCs induced a profound proliferation of alloreactive $\mathrm{CD} 4{ }^{+}$Teff in the presence of autologous Treg, whereas the proliferation of CD4 ${ }^{+}$Teff stimulated by SB202474- or DMSO-treated h-mMoDCs was largely inhibited (Fig. 8c). OX40 costimulation is crucial to p38 MAPK inhibitor-treated h-mMoDCs-mediated activation of Teff in the presence of Treg, because OX40L blockage abrogated this effect (Fig. 8c). Overall, these results highlight the translational potential of inhibition of p38 MAPK during the differentiation of hMoDCs for improving DC-based immunotherapy in human cancers.

\section{Discussion}

p38 MAPK activity is required for LPS-, TNF- $\alpha$ - and CD40Linduced maturation of iDCs ${ }^{35,36}$. However, the present study shows that p38 MAPK activity in DC progenitor cells acts as an antigen presentation attenuator, and disabling this critical brake during DC differentiation endows DCs with superior activity in antigen presentation and induction of immune responses. Our results further demonstrate the crucial role of inhibition of p38 MAPK activity in DCs in activating $\mathrm{CD}^{+}{ }^{+}$and $\mathrm{CD} 4^{+} \mathrm{T}$ cells during both priming and effector phases of the immune response in an antigen-specific manner, possibly owing to the improved maturation status ${ }^{37}$ of mSBDCs as shown by the significantly enhanced expression of MHC class II, CD80, CD86, CD40 and OX40L, as well as IL-12 production. When p38 MAPK signalling in DC progenitor cells was inhibited, the remarkably improved maturation status of mSBDCs was not limited to maturation induced by TNF- $\alpha /$ IL- $1 \beta$, but could also extend to other stimuliinduced maturation, such as LPS, Poly I:C, CpG, sCD40L and 

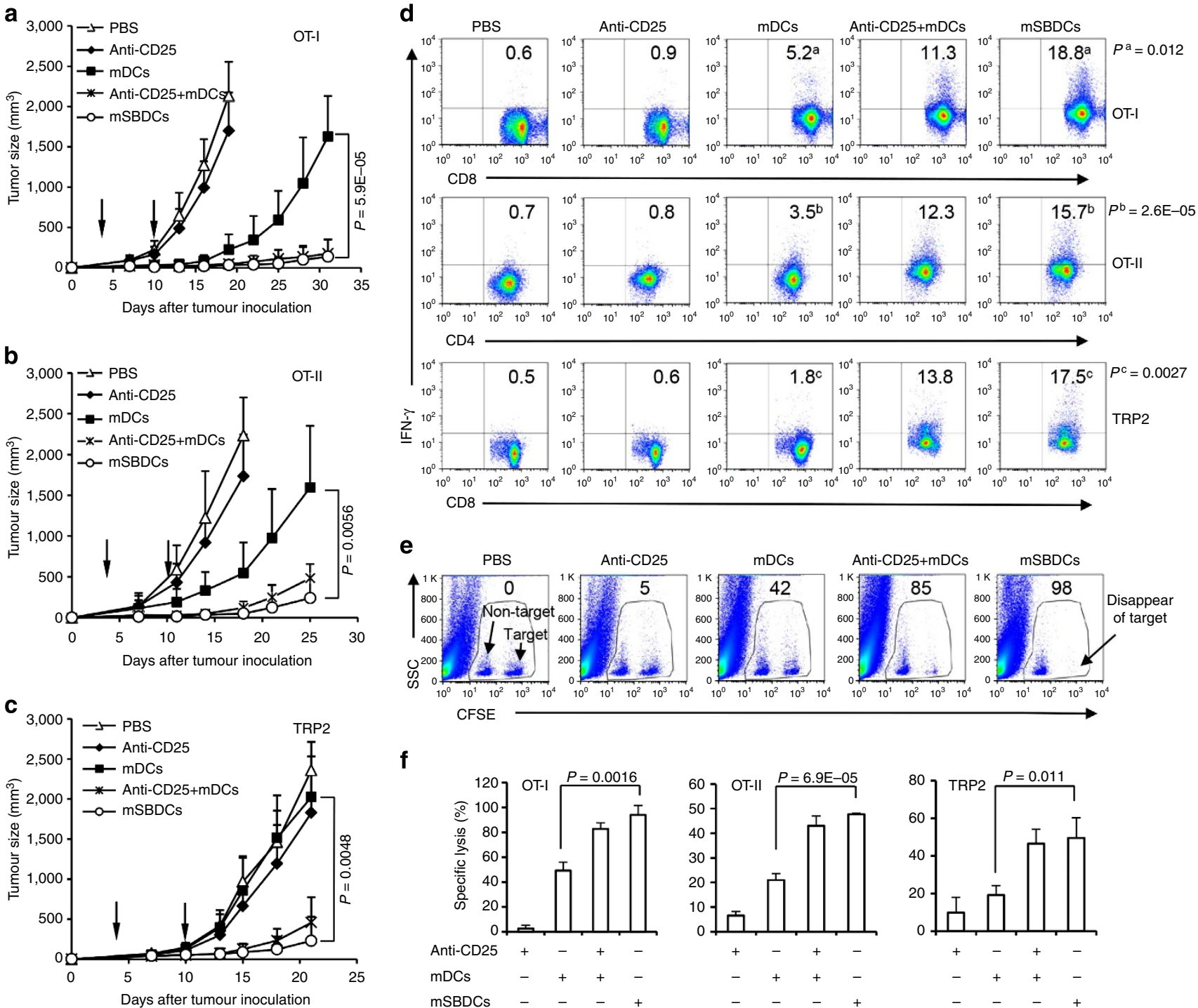

$\mathbf{f}$
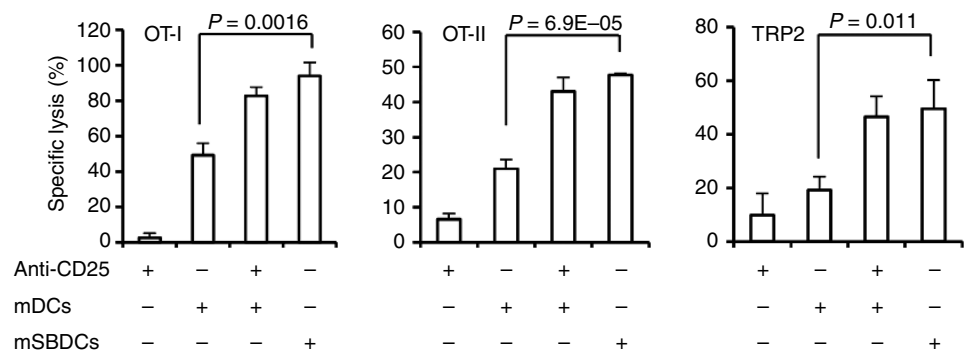

Figure 6 | Antitumour activity of DC immunization and Treg depletion using anti-CD25 antibody. (a-c) Comparison of antitumour activity against pre-established tumours. C57BL/6 mice (five per group) were inoculated subcutaneously with B16-OVA (a,b) or B16 (c) tumour cells ( $3 \times 10^{5}$ ), and 3 days later were immunized with $1 \times 10^{6}$ OT-I peptide-loaded (a), OT-II peptide-loaded (b) or TRP2 peptide-loaded (c) mDCs or mSBDCs twice at a weekly interval. Arrows represent the timing of DC therapy. Anti-CD25 antibodies ( $50 \mu \mathrm{g}$ per mouse) were administered intraperitoneally into some groups of mice 3 days before immunization. Error bars represent s.d. Representative results from one of three repeated experiments are shown. (d) IFN- $\gamma$ ICS of splenocytes from tumour-bearing mice 10 days after second immunization was performed after restimulation with the immunizing peptides overnight in vitro. Numbers represent percentages of IFN- $\gamma$ secreting lymphocytes gated on $\mathrm{CD}^{+}{ }^{+}$or $\mathrm{CD} 8{ }^{+} \mathrm{T}$ cells from one representative experiment of three performed. (e) In vivo CTL assay. CFSE ${ }^{\text {lo }}$ (left population in gates, non-target) and CFSE ${ }^{\text {hi }}$ (right population in gates, target) cells were pulsed with control peptide and OT-I peptide, respectively, and were injected into mice 7 days after OT-I peptide-pulsed DC immunization. Splenocytes were collected $6 \mathrm{~h}$ later for detection of CFSE-labelled cells. Numbers represent percentages of specific killing. Results are representative of one of three independent experiments. (f) Pooled data $(n=3)$ for in vivo CTL assay. Splenocytes were collected $36 \mathrm{~h}$ later for detection of CFSE-labelled cells in OT-II and TRP2 peptide-loaded DC immunization. Error bars represent s.d. $P$ values were calculated with Student's $t$-test.

TNF- $\alpha /$ IL-1 $\beta /$ IL-6/prostaglandin E2 (PGE2; Supplementary Fig. 15). Additional inhibition of p38 MAPK during maturation had no obvious effect in improving SBDC maturation status when matured by TNF- $\alpha / \mathrm{IL}-1 \beta$, sCD $40 \mathrm{~L}$ or TNF- $\alpha / \mathrm{IL}-1 \beta / \mathrm{IL}-6 /$ PGE2, but to some extent, suppressed the maturation of SBDCs stimulated by LPS, Poly I:C or CpG (Supplementary Fig. 15), indicating the important role of p38 MAPK in toll-like receptor (TLR) agonist-induced maturation of iSBDCs. Hence, this study provides new insights into an intracellular negative feedback mechanism, and indicates that DC generation and activation is self-limiting through p38 MAPK activation in DC progenitor cells.
We also found that the self-limiting regulation of DC immunogenicity, controlled by $\mathrm{p} 38 \mathrm{MAPK}$, is mediated through transcriptional repression of NF- $\kappa \mathrm{B}$ family members (Fig. 9). We showed that $\mathrm{p} 38$ MAPK phosphorylates $\mathrm{C} / \mathrm{EBP} \beta$ and $\mathrm{pC} / \mathrm{EBP} \beta$ induces the expression of $\operatorname{PPAR} \gamma$, whose activity affects the maturation and function of $\mathrm{DCs}^{38,39}$. PPAR $\gamma$ has been reported to physically associate with many distinct families of transcription factors, prevent the transcription factors from binding to their response elements and thus suppress their ability to induce gene transcription $^{22}$. In line with this finding, our results revealed a physical interaction between PPAR $\gamma$ and NF- $\kappa \mathrm{B}$ p50, which may repress the transactivation of $\mathrm{p} 50$. Inhibition of $\mathrm{p} 38$ MAPK in 
a

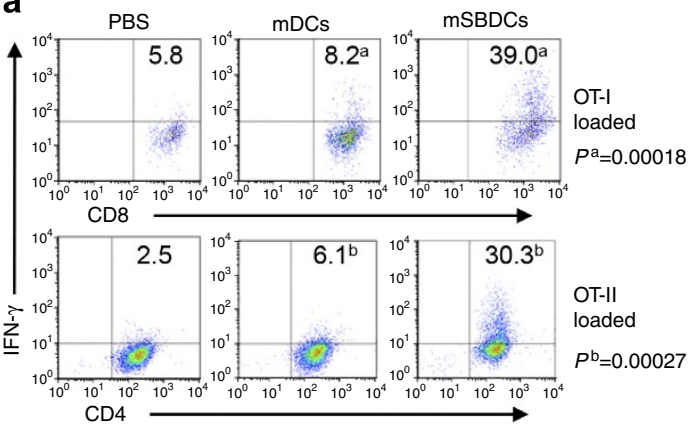

d

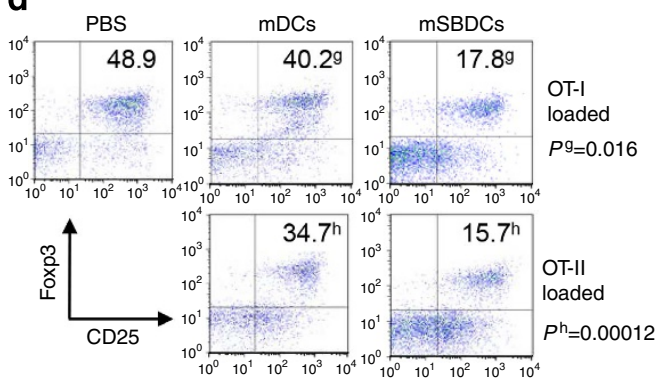

b
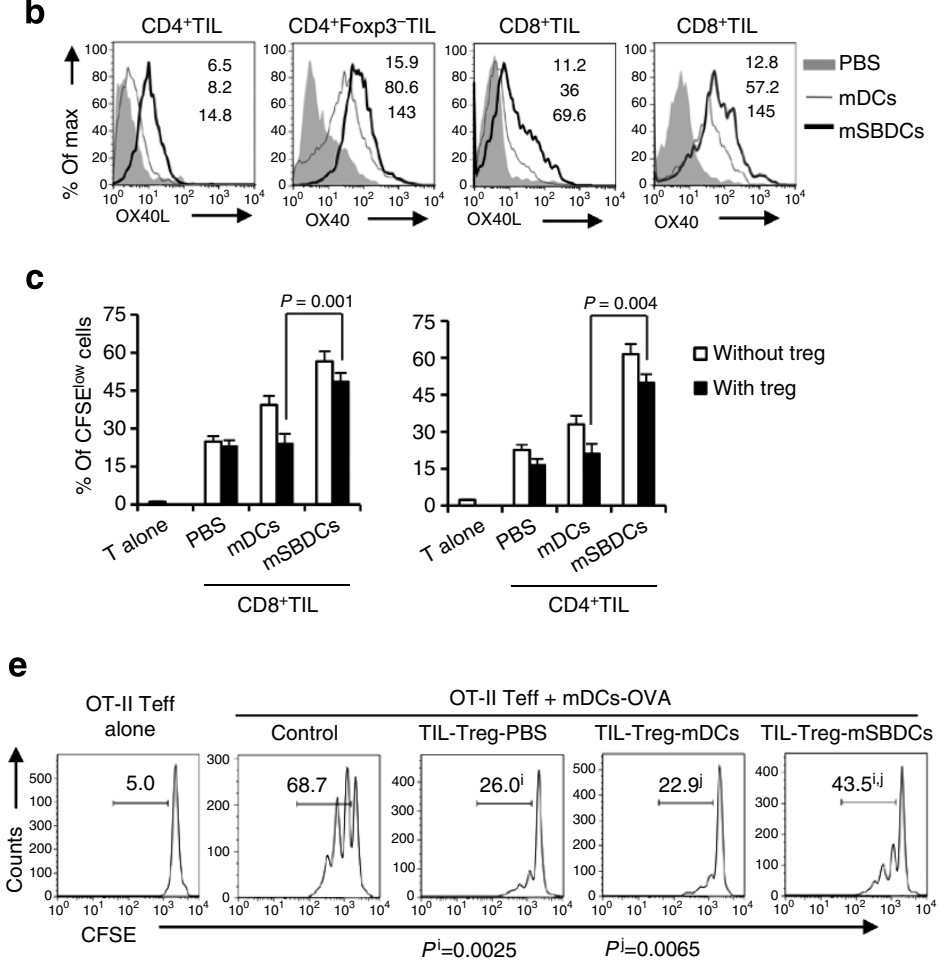

Figure 7 | Effects of $\mathbf{m S B D C}$ on TILs. (a) IFN- $\gamma$ ICS of pooled CD8 ${ }^{+}$T cells or CD4 ${ }^{+}$T cells isolated from TILs of mice 10 days after final immunization was performed after overnight in vitro restimulation with OT-I peptide- or OT-II peptide-loaded mDCs. Numbers represent percentages of IFN- $\gamma$-secreting lymphocytes gated on $\mathrm{CD}^{+}{ }^{+}$or $\mathrm{CD} 8^{+} \mathrm{T}$ cells. (b) Expression (mean fluorescence intensity, MFI) of $\mathrm{OX} 40$ and $\mathrm{OX}_{40 L}$ on $\mathrm{CD}^{+}{ }^{+}$or $\mathrm{CD} 8{ }^{+} \mathrm{T}_{\text {cells }}$ isolated from TILs of mice immunized with OT-II peptide- or OT-I peptide-loaded mDCs and mSBDCs, respectively. (c) Enhanced proliferation of TILs in the absence or presence of Treg. CFSE-labelled $1 \times 10^{5} \mathrm{CD} 8^{+}$Teff (left) or CD4 ${ }^{+} \mathrm{CD} 25^{-}$Teff (right) from TILs were cocultured with $1 \times 10^{4}$ OT-I peptide- or OT-II peptide-loaded mDCs, respectively, in the absence or presence of $1 \times 10^{5}$ unlabelled CD4 ${ }^{+} \mathrm{CD} 25^{+}$Treg from naive mice in triplicates. Error bars represent s.d. $(n=3)$. (d) FACS analysis of the frequency of tumour-infiltrating Treg. Numbers represent the percentages of CD25 ${ }^{+}$Foxp3 ${ }^{+}$Treg in gated CD4 ${ }^{+}$T cells. (e) Reduced suppressive activity of tumour-infiltrating Treg. Splenic CD4 ${ }^{+}$CD25 ${ }^{-}$Teff $\left(1 \times 10^{5}\right)$ from OT-II mice were cocultured with $1 \times 10^{4}$ OVA-loaded mDCs in the absence or presence of CD4 ${ }^{+}$CD $25^{+}$Treg $\left(1 \times 10^{5}\right)$ from TILs. CFSE intensity of Teff was measured by FACS $60 \mathrm{~h}$ later. Numbers indicate percent CFSElo/proliferated Teff. Representative data from one of two (c-e) or three (a,b) repeated experiments are shown. $P$ values were calculated with Student's $t$-test.

DCs abrogated this self-limiting regulation and resulted in a robust p50 nuclear translocation. This sustained activated p50 forms transcriptionally active complexes with RelA, c-Rel and especially with RelB, which bind the $\mathrm{O} x 40 \mathrm{l}$ promoter and induce the substantially increased expression of surface OX40L on mSBDCs. Our result that $p 50$ deficiency abrogated the enhanced expression of OX40L on mSBDCs further supports this conclusion.

Tregs have a major role in tumour-related immune suppression, and have been considered as one of the most crucial hurdles to overcome for successful antitumour immunotherapy ${ }^{40}$. A recent report has shown an attenuated IL-10-secreting Treg induced by TLR agonists through inhibition of p38 MAPK signalling in DCs during maturation, owing to the decreased IL10 production in DCs stimulated with TLR agonists ${ }^{41}$. In addition, others also showed that constitutive activation of $\mathrm{p} 38$ MAPK is responsible for inducing a tolerogenic profile in DCs during melanoma progression in tumour-bearing mice, with DCs secreting significantly more IL-10 and less IL-12 (ref. 42). Indeed, IL-12 might be downregulated by p38 MAPK signalling in many different antigen-presenting cells, especially following TLR agonists stimulation ${ }^{41-45}$. Our findings differ from these reports because we used cytokine cocktails, not TLR agonists, to mDCs; the former are more relevant to the cells that are being used in clinical trials. A surprising effect of mSBDCs in inhibiting conversion of Teff to $\mathrm{CD} 4{ }^{+} \mathrm{CD} 25^{+} \mathrm{Fop} 3{ }^{+}$Treg has been described in this study. The enhanced OX40/OX40L signals provided by mSBDCs may be responsible for the significantly decreased Treg cell population in the tumour and lymph nodes. In line with these result, several groups have reported that the generation of induced Treg is affected by OX40 costimulation $^{25,46}$.

In addition to inhibition of Treg conversion, mSBDCs were more resistant to cancer- and Treg cell-derived suppressive factors (Supplementary Fig. 16). Notably, mSBDCs partially abrogated Treg-mediated immunosuppression by activating $\mathrm{CD} 4{ }^{+}$or $\mathrm{CD} 8{ }^{+}$Teff in the presence of Treg and by inhibiting the suppressive function of Treg, probably via the mechanism of enhanced OX40 signal on both Teff and Treg. Our results show that mSBDCs were as effective as combined treatments of $\mathrm{mDC}$ immunization and Treg depletion by anti-CD25 antibodies in controlling the growth of pre-established tumours. Importantly, we also evidenced profound effects of mSBDCs on the effector phase of antitumour immunity, as demonstrated by substantially increased antigen-specific, activated tumour-infiltrating $\mathrm{CD}^{+}{ }^{+}$or $\mathrm{CD}^{+}{ }^{+}$Teff that were more resistant to Treg-mediated suppression, and by the reduced suppressive function of tumourinfiltrating Treg that may be a result of the increased OX40 signalling delivered by mSBDCs and/or activated Teff within the tumours. OX40L expression is only observed on cells present at sites of inflammation and not in those invading tumours or on tumour cells themselves, and this insufficient ligation of OX40 
a

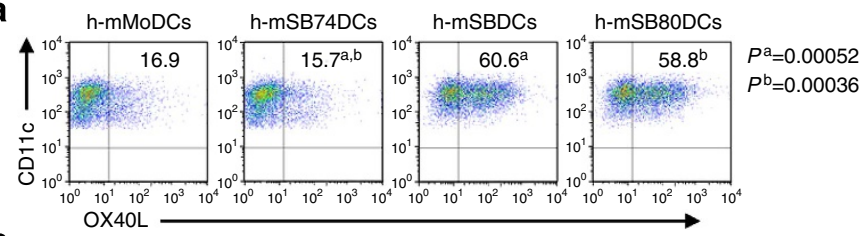

b
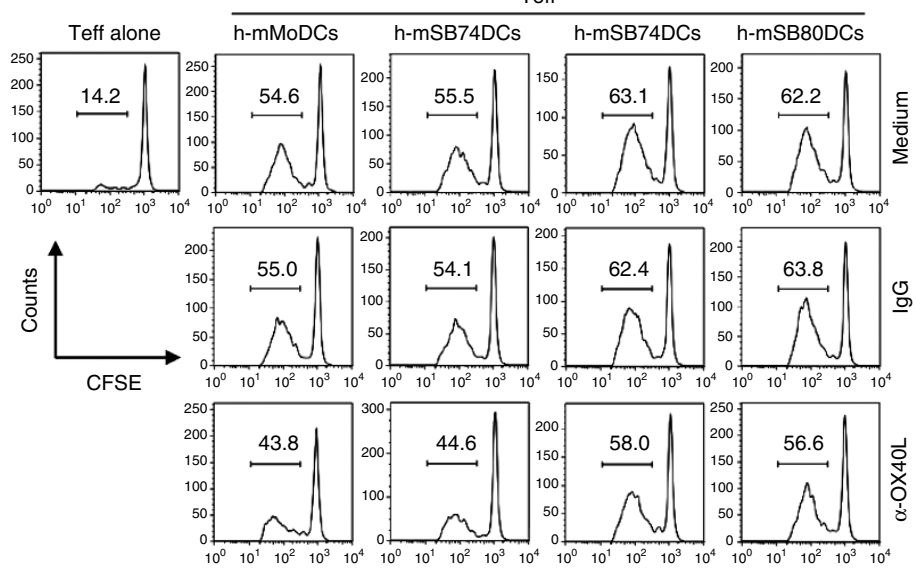

$10^{0} \quad 10^{1} \quad 10^{2} \quad 10^{3} \quad 10^{4}$

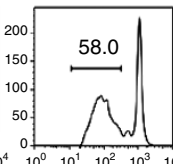

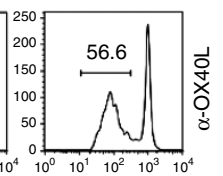

C
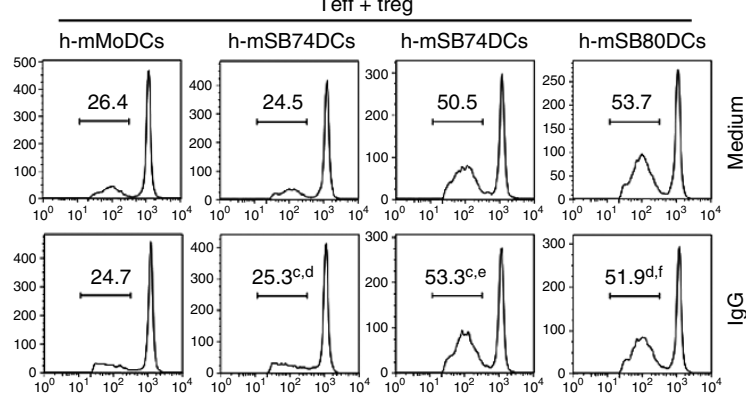

200
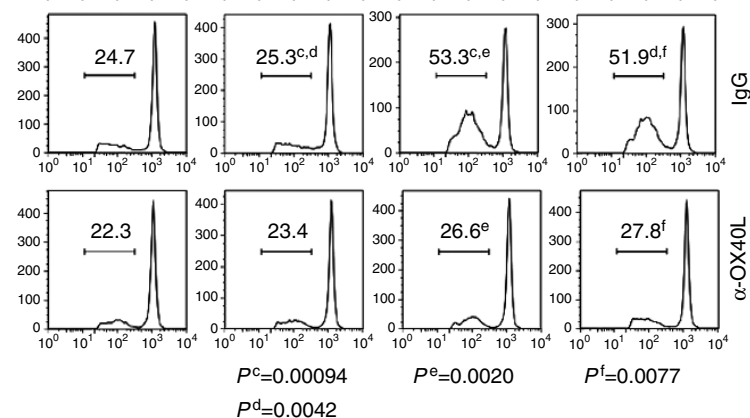

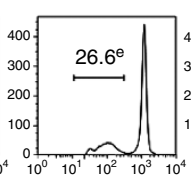

$P^{\mathrm{e}}=0.0020$

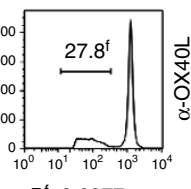

$P^{\mathrm{f}}=0.0077$

Figure 8 | Effects of p38 MAPK inhibition on h-MoDCs. (a) Expression of OX4OL on treated h-mMoDCs. Inhibitors were added during the differentiation of h-MoDCs. Numbers are percentages of OX4OL-expressing CD11c ${ }^{+}$DCs as determined by FACS analysis. (b,c) Proliferation of CFSE-labelled CD4 ${ }^{+}$CD25- Teff $\left(1 \times 10^{5}\right)$ in the absence $(\mathbf{b})$ or presence $(\mathbf{c})$ of unlabelled CD4 ${ }^{+}$CD25 $5^{+}$Treg $\left(1 \times 10^{5}\right)$ induced by various h-mMoDCs $\left(1 \times 10^{4}\right)$. Teff and Treg were isolated from different donors of peripheral blood mononuclear cells. Treg and h-mMoDCs were derived from the same donors. Control lgG or anti-OX4OL mAbs were added to the coculture. Six days later, CFSE intensity of Teff was measured by FACS. Numbers indicate percent CFSElo/ proliferated Teff. Representative results from one of two performed experiments are shown. $P$ values were calculated with Student's $t$-test.

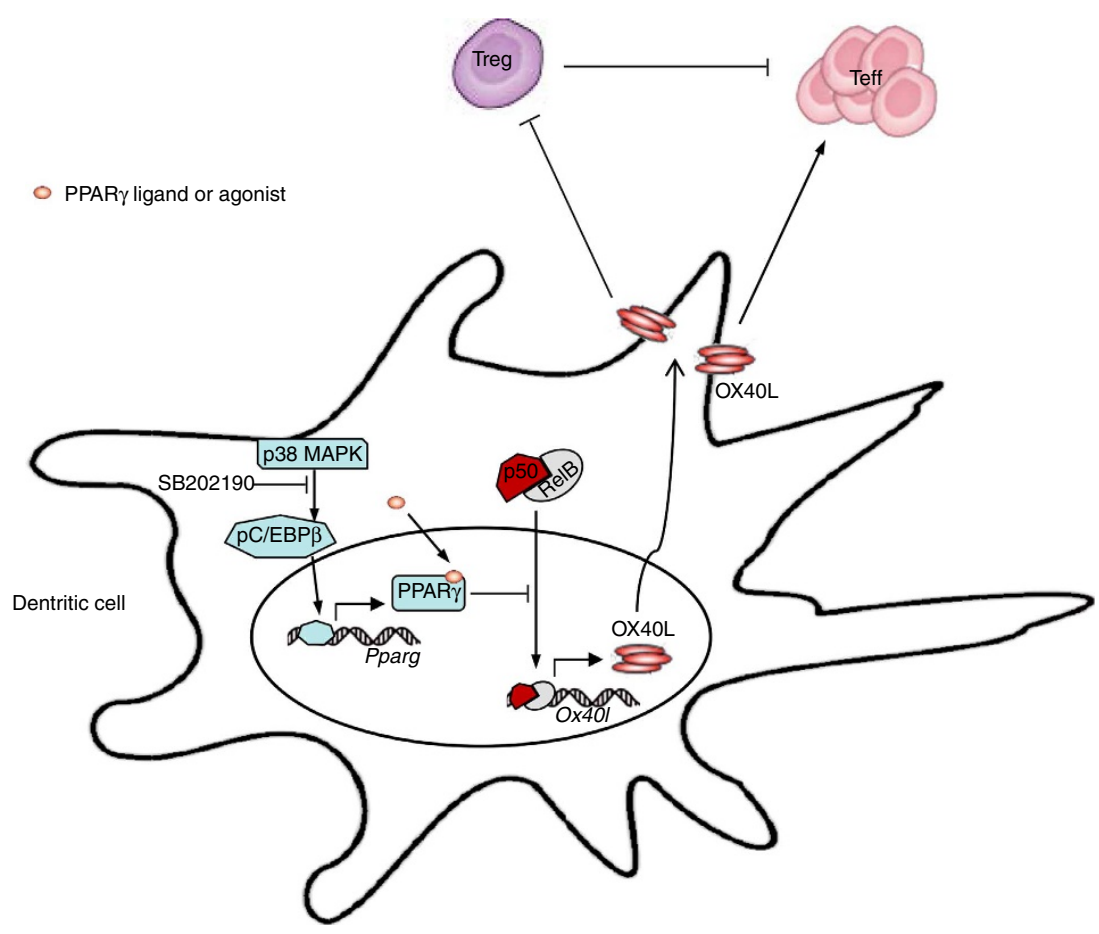

Figure 9 | The role of $\mathbf{p} 38$ MAPK signalling in the regulation of DC immunogenicity. $p 38$ MAPK phosphorylates $C / E B P \beta$, and $p C / E B P \beta$ induces the production of PPAR $\gamma$. PPAR $\gamma$ can be activated by its ligand or agonist that can then repress the transactivation of p50 by physical interaction with p50 to prevent its binding to the response elements such as Ox40/ promoter. p38 MAPK inhibitor, such as SB202190, could abrogate this self-limitation break and result in a robust p50 nuclear translocation. p50, after forming transcriptionally active complexes with RelB, induces the strikingly increased surface OX40L expression, which is crucial to activation of Teff and inhibition of Treg. 
could be a limiting factor for immune-mediated destruction of tumour cells ${ }^{47}$. In support of our findings, OX40L expressed by mDCs has been shown to be important for induction of antitumour immunity ${ }^{48}$, and other groups also demonstrated that OX40 signalling triggered by agonist antibodies facilitates tumour rejection by blocking the suppression of Treg ${ }^{49}$. Taken together, in addition to its strong immunostimulatory effects on Teff, mSBDCs can induce efficient antitumour immune responses by counteracting Treg-mediated immune suppression. Hence, mSBDCs should be very useful as a tool for DC-based immunotherapy to induce strong antitumour immune responses in cancer patients.

\section{Methods}

Mice. C57BL/6 mice were purchased from the National Cancer Institute (NCI), and OX40-I- (B6.129S4-Tnfrsf4 ${ }^{\text {tm1Nik} / J), ~ O T-I I ~(C 57 B L / 6-T g(T c r a T c r b) 425 C b n / J), ~}$ OT-I (C57BL/6-Tg(TcraTcrb) $1100 \mathrm{Mjb} / \mathrm{J})$ and $p 50^{-1-}$ (C57BL/6-129P$\left.\mathrm{Nfkb}^{\mathrm{tm} 1 \mathrm{Bal}} / \mathrm{J}\right)$ mice were purchased from the Jackson Laboratory. Male, 6-8-week old mice were used in the beginning of each animal experiment. The studies were approved by the Institutional Animal Care and Use Committee of Cleveland Clinic Foundation.

Cell lines and reagents. The WT B16 and B16 transfected with OVA (B16-OVA) melanoma cell lines (American Type Culture Collection (ATCC)) were cultured in Iscove's modified Dulbecco's media (Invitrogen) supplemented with $10 \%$ heatinactivated fetal bovine serum (FBS; Thermo Scientific), $100 \mathrm{U} \mathrm{ml}^{-1}$ penicillinstreptomycin and $2 \mathrm{mM}$ L-glutamine (both from Invitrogen). p38 MAPK inhibitor SB202190 and SB203580, and their inactive analogue SB202474 were purchased from EMD Biosciences. PGE2, p38 MAPK activator anisomycin, PPAR $\gamma$ activator RGZ and PPAR $\gamma$ inhibitor GW9662 were purchased from Sigma. MHC class IIrestricted TRP1 (SGHNCGTCRPGWRGAACNQKILTVR), H2-K ${ }^{\mathrm{b}}$-restricted TRP2 (SVYDFFVWL), H2-K ${ }^{\mathrm{b}}$-restricted OT-I (SIINFEKL) and MHC class IIrestricted OT-II (ISQAVHAAHAEINEAGR) peptides were purchased from GenScript. Murine OX40L-blocking antibody (RM134L), CD40-blocking antibody (160402), CD80-blocking antibody (16-10A1), CD86-blocking antibody (GL1), IL-12blocking antibody (C17.8) and anti-CD25 antibody (PC6.1) were purchased from eBioscience. Human OX40L-blocking antibody (159403) was purchased from R\&D Systems. Mouse or human Granulocyte-macrophage colony-stimulating factor (GM-CSF), TNF- $\alpha$, IL-1 $\beta$, IL-4, IL-6, IL-2, sCD40L and human TNF- $\beta 1$ were purchased from R\&D Systems. LPS, CpG, Poly I:C were purchased from InvivoGen.

BMDC generation. Mouse BMDCs were prepared as described previously ${ }^{50}$. In brief, BM cells were cultured with $2.5 \mathrm{ml}$ of RPMI-1640 supplemented with $10 \%$ FBS and mGM-CSF $\left(20 \mathrm{ng} \mathrm{ml}^{-1}\right)$. At day 4, $2.5 \mathrm{ml}$ fresh medium containing mGM-CSF $\left(20 \mathrm{ng} \mathrm{ml}^{-1}\right)$ was added. At day 7 , semi-adherent cells were collected as iDCs. In some cultures, $1.5 \mu \mathrm{M}$ of SB202190, SB203580 or SB202474 was added to the cultures on day 0 , and cultures with addition of DMSO $(0.1 \%$; Sigma) served as control. iDCs collected at day 7 were extensively washed and maintained in medium containing mGM-CSF $\left(10 \mathrm{ng} \mathrm{ml}^{-1}\right)$ before adding TNF- $\alpha\left(10 \mathrm{ng} \mathrm{ml}^{-1}\right)$ and IL-1 $\beta\left(10 \mathrm{ng} \mathrm{ml}^{-1}\right)$ to help mature the cells. After $48 \mathrm{~h}$, semi-adherent cells were collected as mDCs. No additional DMSO, SB202190, SB203580 or SB202474 was added during the culture or during maturation. Flow cytometry analysis showed that both iDCs and mDCs contain $>90 \%$ of CD11c ${ }^{+}$cells, while mDCs express significantly upregulated surface expression of DC-related molecules than iDCs (Supplementary Fig. 1).

h-MoDC generation. h-MoDCs were generated as described previously ${ }^{51}$. Monocytes were positively selected from peripheral blood mononuclear cells of healthy donors using anti-CD14-conjugated magnetic microbeads (Miltenyi Biotec). Monocytes were cultured in AIM-V medium supplemented with 50 ng ml ${ }^{-1}$ human GM-CSF and human Interleukin 4 (hIL-4). In some cultures, $1 \mu \mathrm{M}$ of SB202190, SB203580 (ref. 52) or SB202474 was added to the cultures on day 0 . After 5 days, semi-adherent cells iDCs were extensively washed and then matured for 2 days by adding human tumor necrosis factor- $\alpha$ (hTNF- $\alpha$ ) $\left(10 \mathrm{ng} \mathrm{ml}^{-1}\right)$, hIL-1 $\beta\left(10 \mathrm{ng} \mathrm{ml}^{-1}\right)$, hIL-6 $\left(10 \mathrm{ng} \mathrm{ml}^{-1}\right)$ and $0.5 \mu \mathrm{g} \mathrm{ml}^{-1}$ PGE2. The study was approved by the Institutional Review Board Committee of Cleveland Clinic Foundation.

Flow cytometric analysis. Cells were stained with fluorescein isothiocyanate (FITC), phycoerythrin, (PE), allophycocyanin (APC)-conjugated mAbs (1:100 dilution) after pre-blocking FC $\gamma$ receptors. Rat mAbs specific for mouse CD4 (GK1.5), CD8 (53-6.7), CD25 (PC6.1), CD11c (N418), CD40 (1C10), CD80 (16-10A1), CD86 (GL1), OX40L (RM134L), OX40 (OX86) and matched isotype controls were purchased from eBioscience. Intracellular IFN- $\gamma$ staining for T cells was performed using a Cytofix/Cytoperm kit (BD Biosciences), with anti-IFN- $\gamma$ (XMG1.2) antibodies (eBioscience). Before intracellular IFN- $\gamma$ staining, splenocytes were restimulated by peptides $\left(5 \mu \mathrm{g} \mathrm{ml}^{-1}\right)$ or peptide-loaded mDCs at a 10:1 $\mathrm{T}$ cells to DCs ratio overnight, and for the final $6 \mathrm{~h}$ of culture, GolgiPlug (BD Biosciences-Pharmingen) was added. Foxp3 staining was performed using a Foxp3 staining kit (eBioscience) with anti-Foxp3 (FJK-16s) antibodies (eBioscience). For flow cytometric analysis, stained cells were analysed on a FACSCalibur (Becton Dickinson) and CELLQuest software.

In vivo CTL assay. In vivo CTL assay was performed as described previously ${ }^{53}$ with some modifications. Spleen cells from C57BL/6 mice were pulsed with $5 \mu \mathrm{g} \mathrm{ml}^{-1}$ OT-I peptide, OT-II peptide, TRP1 peptide or TRP2 peptide. Pulsed splenocytes were labelled with CFSE at a final concentration of $1 \mu \mathrm{M}\left(\mathrm{CFSE}^{\mathrm{hi}}\right)$ or at a final concentration of $0.1 \mu \mathrm{M}\left(\mathrm{CFSE}^{\mathrm{lo}}\right)$. Two cell populations were mixed at $1: 1$ ratio, and $2 \times 10^{7}$ total cells were injected intravenously into treated mice. The percentage of antigen-specific lysis was determined $6 \mathrm{~h}$ later after target cell injection in OT-I peptide-loaded DC-immunized mice (OT-I peptide-pulsed CFSE $^{\text {hi }}$ target + TRP2 peptide-pulsed CFSE ${ }^{\text {lo }}$ non-target), $36 \mathrm{~h}$ later in TRP2 peptide-loaded DC-immunized mice (TRP2 peptide-pulsed CFSE ${ }^{\text {hi }}$ target + OT-1 peptide-pulsed CFSE ${ }^{10}$ non-target), and $36 \mathrm{~h}$ later in OT-II peptide-loaded DCimmunized mice (OT-II peptide-pulsed CFSE ${ }^{\text {hi }}$ target + TRP1 peptide-pulsed CFSE $^{\text {lo }}$ non-target), by analysing the relative proportion of CFSE ${ }^{\text {hi }}$ target cells and $\mathrm{CFSE}^{\text {lo }}$ non-target cells in splenocytes based on CFSE-labeling intensity. The percentage of specific lysis was calculated as follows: $(1-\mathrm{R} \times \mathrm{M} 2 / \mathrm{M} 1) \times 100$, where $\mathrm{M} 1=$ non-target events, $\mathrm{M} 2=$ target events, $\mathrm{R}=\mathrm{M} 1$ in untreated mice/ M2 in untreated mice.

Isolation of TILs. On different days after DC immunization, mice were killed and TILs were isolated from tumour cell suspensions after a Ficoll gradient centrifugation to eliminate dead cells. Subsets of TILs $\left(\mathrm{CD} 8{ }^{+}, \mathrm{CD}^{+}{ }^{+} \mathrm{CD} 25^{-}\right.$and $\mathrm{CD} 4{ }^{+} \mathrm{CD} 25^{+} \mathrm{T}$ cells) were sorted with flow cytometer after staining.

Enzyme-linked immunosorbent assay and western blot. Enzyme-linked immunosorbent assay for IFN- $\gamma$ and IL-12p70 production was performed by ELISA kits (R\&D Systems). For western blot, anti-mouse p38 $\alpha$ (cat. no. 9218), p38 $\gamma$ (cat. no. 2307), p38 (cat. no. 8690), p-p38 (cat. no. 4631), MAPKAPK-2 (cat. no. 3042), pMAPKAPK-2 (cat. no. 3041), RelA (cat. no. 4764), RelB (cat. no. 4954), p52 (cat. no. 4882), GAPDH (cat. no. 2118), PARP (cat. no. 9532), C/EBP- $\beta$ (cat. no. 3087), pC/EBP- $\beta$ (cat. no. 3084), PPAR $\gamma$ (cat. no. 2430), pStat3 (cat. no. 9145), Stat3 (cat. no. 12640), pSmad3 (cat. no. 9520), Smad3 (cat. no. 9523), IкB- $\alpha$ (cat. no. 4812$)$ and $\beta$-actin (cat. no. 4970) from Cell Signaling (1:1,000 dilution), and anti-mouse p38 $\beta$, (cat. no. sc-6187), p38 , (cat. no. sc-7587), p50 (cat. no. sc-7178) and c-Rel (cat. no. sc-70) from Santa Cruz Biotechnology (1:250 dilution) were used.

T-cell proliferation and suppression assays. Splenic $\mathrm{CD} 8{ }^{+}, \mathrm{CD} 4{ }^{+} \mathrm{CD} 25^{-}$or $\mathrm{CD} 4{ }^{+} \mathrm{CD} 25^{+} \mathrm{T}$ cells from WT, OX40 ${ }^{-1-}$, OT-I- or OT-II-transgenic mice were isolated by using the MACS T-cell isolation kits (Miltenyi Biotec). T-cell proliferation was measured with CFSE dilution assay. CD8 ${ }^{+}$or CD4 ${ }^{+} \mathrm{CD} 25^{-} \mathrm{T}$ cells were incubated for $5 \mathrm{~min}$ at $37^{\circ} \mathrm{C}$ with $1 \mu \mathrm{M}$ CFSE in PBS and then washed extensively. For antigenic stimulation using TCR-transgenic OT-I or OT-II T cells and antigen-pulsed DCs, $1 \times 10^{5}$ purified T cells and $1 \times 10^{4}$ DCs pulsed with OT-I or OT-II peptides $\left(1 \mu \mathrm{g} \mathrm{ml}^{-1}\right)$ were seeded in each well of a round-bottom 96 -well microtiter plate in triplicate in $200 \mathrm{ml}$ RPMI-1640 medium supplemented with $10 \%$ FBS. For stimulation using WT or OX40 ${ }^{-1-} \mathrm{T}$ cells, $1 \times 10^{5}$ purified $\mathrm{T}$ cells and $1 \times 10^{4}$ unpulsed DCs were cocultured in the presence of anti-CD3 antibodies $\left(1 \mu \mathrm{g} \mathrm{ml}^{-1}\right)$. For suppression assays, $1-2 \times 10^{5}$ unlabelled $\mathrm{CD} 4{ }^{+} \mathrm{CD} 25^{+}$Tregs were added in the cocultures. Isotype-matched control immunoglobulin G (IgG) and blocking mAbs were added at a concentration of $10 \mu \mathrm{g} \mathrm{ml}^{-1}$. We measured proliferation by relative CFSE dilution after $60 \mathrm{~h}$ of culture. For human T-cell proliferation, h-MoDCs were mixed with CFSE-labelled alloreactive CD4 ${ }^{+} \mathrm{CD} 25^{-}$ Teff in Aim-V medium in the presence or absence of autologous CD4 ${ }^{+} \mathrm{CD} 25^{+}$ Treg. Human T-cell proliferation was assessed 6 days after coculture.

Chromatin immunoprecipitation. ChIP assay were performed with the ChIP assay kit (Millipore) according to the manufacturer's instructions. Chromatin was extracted from $\mathrm{CD} 4{ }^{+}$Foxp $3^{-} \mathrm{T}$ cells cocultured for 4 days with $\mathrm{mDCs}$ or mSBDCs in the presence of TGF- $\beta 1, \mathrm{mDCs}$ and $\mathrm{mSBDCs}$ after fixation with formaldehyde. Anti-pSmad3, anti-pStat3, anti-RelA, anti-RelB, anti-p50 and isotype-matched control antibodies (all from Cell Signaling, 1:20 dilution) were used for the immunoprecipitation of chromatin. The precipitated DNA was analysed by reverse transcription (RT)-PCR with the following primer sets: surrounding region of NF- $\kappa \mathrm{B}$ binding site at $\mathrm{O} x 40 \mathrm{l}$ promoter, $5^{\prime}$-CTTCAGAGCACACGTTTGTG AAT- $3^{\prime}$ and $5^{\prime}$-TGAAGG AGCAGAGCAGAGTCTCTT-3'; enhancer I region: $5^{\prime}$-caggctgacctcaaactcacaaag- $3^{\prime} ; 5^{\prime}$-catacccacactttgacctctgc- $3^{\prime} ; 5^{\prime}$-gcttctgtgtatggttt gtgt- $3^{\prime} ; 5^{\prime}$-atcatcacagtacatacgagga- $3^{\prime}$; enhancer II region: $5^{\prime}$-GGACGTCACCTACC ACATCC-3'; 5'-CTACCCCACAGGTTTCGTTC-3'; $5^{\prime}$-AGAACTTGGGTTTTG CATGG-3'; $5^{\prime}$-TCTACCCCACAGGTTTCGTT- $3^{\prime}$. Values were subtracted with the amount from IgG control and were normalized to corresponding input control. 
Luciferase reporter assays. HEK $293 \mathrm{~T}$ cells were transiently transfected with a 1121-bp mouse $\mathrm{Ox} 40 \mathrm{l}$ promoter (Genecopoeia)-inserted luciferase reporter vector pEZX-PG04 (mOx401-PG40), or control vector (NEG-PG40) and expression vectors for NF- $\mathrm{KB}$ molecules (p50, p52, RelA, RelB and c-Rel, Addgene) by Lipofectamine 2000 (Invitrogen). Promoter activity was measured with SecretePair Dual Luminescence Assay Kit (GeneCopoeia) according to the manufacturer's instructions. Values are expressed as the mean \pm s.d. of relative luciferase units normalized to internal control.

DC immunization and tumour models. iDCs, iSBDCs, $\mathrm{p} 50^{-/-}$iSBDCs, iSB74DCs, iSB80DCs and siRNA-treated iDCs were pulsed with OT-I $\left(5 \mu \mathrm{g} \mathrm{ml}^{-1}\right)$, OT-II $\left(15 \mu \mathrm{g} \mathrm{ml}^{-1}\right)$ or TRP2 peptides $\left(30 \mu \mathrm{g} \mathrm{ml}^{-1}\right)$ for $8 \mathrm{~h}$, and matured for additional $48 \mathrm{~h}$ in the presence of TNF- $\alpha$ and IL-1 $\beta$. After washing with PBS, $\mathrm{mDCs}$ or treated $\mathrm{mDCs}$ were prepared for immunization of mice (five per group). In the therapeutic model, $3 \times 10^{5} \mathrm{~B} 16$-OVA or B16 tumour cells were injected subcutaneously into the right flank of WT C57BL/6 mice. Mice were then randomly divided into groups and injected with $1 \times 10^{6} \mathrm{mDCs}$ or treated $\mathrm{mDCs}$ subcutaneously in the left back 3 and 10 days later. Tumour volumes were measured two or three times per week with an electronic caliper.

Statistical analyses. For statistical analysis, Student's $t$-test was used. A $P$ value $<0.05$ was considered statistically significant. In some Figures, the actual $P$ values were shown as superscript numbers. Results are typically presented as means \pm s.d.

\section{References}

1. Kalinski, P., Muthuswamy, R. \& Urban, J. Dendritic cells in cancer immunotherapy: vaccines and combination immunotherapies. Expert Rev. Vaccines 12, 285-295 (2013).

2. Heiro, Y. i. D. \& Appel, S. Current status and future perspectives of dendritic cell-based cancer immunotherapy. Scand. J. Immunol. 78, 167-171 (2013).

3. Zou, W. Regulatory T cells, tumour immunity and immunotherapy. Nat. Rev. Immunol. 6, 295-307 (2006).

4. Menetrier-Caux, C., Gobert, M. \& Caux, C. Differences in tumor regulatory T-cell localization and activation status impact patient outcome. Cancer Res. 69, 7895-7898 (2009).

5. Zou, W. Immunosuppressive networks in the tumour environment and their therapeutic relevance. Nat. Rev. Cancer 5, 263-274 (2005).

6. Feng, L. L. \& Wang, X. Targeting Foxp3 + regulatory T cells-related immunosuppression for cancer immunotherapy. Chin. Med. J. (Engl) 123, 3334-3342 (2010).

7. Byrne, W. L., Mills, K. H., Lederer, J. A. \& O’Sullivan, G. C. Targeting regulatory T cells in cancer. Cancer Res. 71, 6915-6920 (2011).

8. Shimizu, J., Yamazaki, S., Takahashi, T., Ishida, Y. \& Sakaguchi, S. Stimulation of $\mathrm{CD} 25(+) \mathrm{CD} 4(+)$ regulatory $\mathrm{T}$ cells through GITR breaks immunological self-tolerance. Nat. Immunol. 3, 135-142 (2002).

9. Piconese, S., Valzasina, B. \& Colombo, M. P. OX40 triggering blocks suppression by regulatory $\mathrm{T}$ cells and facilitates tumor rejection. J. Exp. Med. 205, 825-839 (2008).

10. Valzasina, B., Piconese, S., Guiducci, C. \& Colombo, M. P. Tumor-induced expansion of regulatory $\mathrm{T}$ cells by conversion of $\mathrm{CD} 4+\mathrm{CD} 25$ - lymphocytes is thymus and proliferation independent. Cancer Res. 66, 4488-4495 (2006).

11. Ramirez-Montagut, T. et al. Glucocorticoid-induced TNF receptor family related gene activation overcomes tolerance/ignorance to melanoma differentiation antigens and enhances antitumor immunity. J. Immunol. 176, 6434-6442 (2006).

12. Cohen, A. D. et al. Agonist anti-GITR antibody enhances vaccine-induced CD8(+) T-cell responses and tumor immunity. Cancer Res. 66, 4904-4912 (2006).

13. Attia, P. et al. Autoimmunity correlates with tumor regression in patients with metastatic melanoma treated with anti-cytotoxic T-lymphocyte antigen-4. J. Clin. Oncol. 23, 6043-6053 (2005).

14. Xie, J. et al. Novel and detrimental effects of lipopolysaccharide on in vitro generation of immature dendritic cells: involvement of mitogen-activated protein kinase p38. J. Immunol. 171, 4792-4800 (2003).

15. Wang, S. et al. Optimizing immunotherapy in multiple myeloma: restoring the function of patients' monocyte-derived dendritic cells by inhibiting p38 or activating MEK/ERK MAPK and neutralizing interleukin-6 in progenitor cells. Blood 108, 4071-4077 (2006).

16. Wang, S. et al. Tumor evasion of the immune system: inhibiting p38 MAPK signaling restores the function of dendritic cells in multiple myeloma. Blood 107, 2432-2439 (2006).

17. Oosterhoff, D. et al. Tumor-mediated inhibition of human dendritic cell differentiation and function is consistently counteracted by combined p38 MAPK and STAT3 inhibition. Oncoimmunology 1, 649-658 (2012).
18. Cirone, M. et al. Dendritic cell differentiation blocked by primary effusion lymphoma-released factors is partially restored by inhibition of P38 MAPK. Int. J. Immunopathol. Pharmacol. 23, 1079-1086 (2010).

19. Lee, J. C. et al. A protein kinase involved in the regulation of inflammatory cytokine biosynthesis. Nature 372, 739-746 (1994).

20. Tabuchi, K. et al. Cochlear protection from acoustic injury by inhibitors of p38 mitogen-activated protein kinase and sequestosome 1 stress protein. Neuroscience 166, 665-670 (2010).

21. Croft, M. Control of immunity by the TNFR-related molecule OX40 (CD134), Annu. Rev. Immunol. 28, 57-78 (2010).

22. Takeda, I. et al. Distinct roles for the OX40-OX40 ligand interaction in regulatory and nonregulatory T cells. J. Immunol. 172, 3580-3589 (2004).

23. Daynes, R. A. \& Jones, D. C. Emerging roles of PPARs in inflammation and immunity. Nat. Rev. Immunol. 2, 748-759 (2002).

24. Watts, T. H. TNF/TNFR family members in costimulation of T cell responses. Annu. Rev. Immunol. 23, 23-68 (2005).

25. Jensen, S. M. et al. Signaling through OX40 enhances antitumor immunity. Semin. Oncol. 37, 524-532 (2010).

26. So, T. \& Croft, M. Cutting edge: OX40 inhibits TGF-beta- and antigen-driven conversion of naive CD4 T cells into CD25 + Foxp3 + T cells. J. Immunol. 179, 1427-1430 (2007).

27. Xu, L. et al. Positive and negative transcriptional regulation of the Foxp3 gene is mediated by access and binding of the Smad3 protein to enhancer I. Immunity 33, 313-325 (2010).

28. Trempolec, N., Dave-Coll, N. \& Nebreda, A. R. SnapShot: p38 MAPK signaling. Cell 152, 656-656el (2013).

29. Wu, Z., Bucher, N. L. \& Farmer, S. R. Induction of peroxisome proliferatoractivated receptor gamma during the conversion of $3 \mathrm{~T} 3$ fibroblasts into adipocytes is mediated by C/EBPbeta, C/EBPdelta, and glucocorticoids. Mol. Cell. Biol. 16, 4128-4136 (1996).

30. Szatmari, I., Rajnavolgyi, E. \& Nagy, L. PPARgamma, a lipid-activated transcription factor as a regulator of dendritic cell function. Ann. NY Acad. Sci. 1088, 207-218 (2006).

31. He, J. et al. p38 MAPK in myeloma cells regulates osteoclast and osteoblast activity and induces bone destruction. Cancer Res. 72, 6393-6402 (2012).

32. Song, X. T. et al. A20 is an antigen presentation attenuator, and its inhibition overcomes regulatory T cell-mediated suppression. Nat. Med. 14, 258-265 (2008).

33. Evel-Kabler, K., Song, X. T., Aldrich, M., Huang, X. F. \& Chen, S. Y. SOCS1 restricts dendritic cells' ability to break self tolerance and induce antitumor immunity by regulating IL-12 production and signaling. J. Clin. Invest. 116, 90-100 (2006).

34. Prasad, S. J. et al. Dendritic cells loaded with stressed tumor cells elicit longlasting protective tumor immunity in mice depleted of CD4 + CD25 + regulatory T cells. J. Immunol. 174, 90-98 (2005).

35. Arrighi, J. F., Rebsamen, M., Rousset, F., Kindler, V. \& Hauser, C. A critical role for p38 mitogen-activated protein kinase in the maturation of human bloodderived dendritic cells induced by lipopolysaccharide, TNF-alpha, and contact sensitizers. J. Immunol. 166, 3837-3845 (2001).

36. Yu, Q., Kovacs, C., Yue, F. Y. \& Ostrowski, M. A. The role of the p38 mitogenactivated protein kinase, extracellular signal-regulated kinase, and phosphoinositide-3-OH kinase signal transduction pathways in CD40 ligandinduced dendritic cell activation and expansion of virus-specific CD8 + T cell memory responses. J. Immunol. 172, 6047-6056 (2004).

37. Villadangos, J. A. \& Schnorrer, P. Intrinsic and cooperative antigen-presenting functions of dendritic-cell subsets in vivo. Nat. Rev. Immunol. 7, 543-555 (2007).

38. Szatmari, I. et al. PPARgamma regulates the function of human dendritic cells primarily by altering lipid metabolism. Blood 110, 3271-3280 (2007).

39. Kock, G. et al. Regulation of dectin-1-mediated dendritic cell activation by peroxisome proliferator-activated receptor-gamma ligand troglitazone. Blood 117, 3569-3574 (2011).

40. Zitvogel, L., Tesniere, A. \& Kroemer, G. Cancer despite immunosurveillance: immunoselection and immunosubversion. Nat. Rev. Immunol. 6, 715-727 (2006).

41. Jarnicki, A. G. et al. Attenuating regulatory T cell induction by TLR agonists through inhibition of p38 MAPK signaling in dendritic cells enhances their efficacy as vaccine adjuvants and cancer immunotherapeutics. J. Immunol. 180, 3797-3806 (2008).

42. Zhao, F. et al. Activation of p38 mitogen-activated protein kinase drives dendritic cells to become tolerogenic in ret transgenic mice spontaneously developing melanoma. Clin. Cancer Res. 15, 4382-4390 (2009).

43. Yang, Z., Zhang, X., Darrah, P. A. \& Mosser, D. M. The regulation of Th1 responses by the p38 MAPK. J. Immunol. 185, 6205-6213 (2010).

44. Franks, H. A. et al. Novel function for the p38-MK2 signaling pathway in circulating CD1c $+($ BDCA-1 +$)$ myeloid dendritic cells from healthy donors and advanced cancer patients; inhibition of p38 enhances IL-12 whilst suppressing IL-10. Int. J. Cancer 134, 575-586 (2014). 
45. Kuhnol, C., Herbarth, M., Foll, J., Staege, M. S. \& Kramm, C. CD137 stimulation and 38 MAPK inhibition improve reactivity in an in vitro model of glioblastoma immunotherapy. Cancer Immunol. Immunother. 62, 1797-1809 (2013).

46. Vu, M. D. et al. OX40 costimulation turns off Foxp3 + Tregs. Blood 110, 2501-2510 (2007).

47. Gough, M. J. \& Weinberg, A. D. OX40 (CD134) and OX40L. Adv. Exp. Med. Biol. 647, 94-107 (2009).

48. Zaini, J. et al. OX40 ligand expressed by DCs costimulates NKT and CD4 + Th cell antitumor immunity in mice. J. Clin. Invest. 117, 3330-3338 (2007).

49. Moran, A. E., Kovacsovics-Bankowski, M. \& Weinberg, A. D. The TNFRs OX40, 4-1BB, and CD40 as targets for cancer immunotherapy. Curr. Opin. Immunol. 25, 230-237 (2013).

50. Hong, S. et al. Optimizing dendritic cell vaccine for immunotherapy in multiple myeloma: tumour lysates are more potent tumour antigens than idiotype protein to promote anti-tumour immunity. Clin. Exp. Immunol. 170, 167-177 (2012).

51. Legler, D. F., Krause, P., Scandella, E., Singer, E. \& Groettrup, M. Prostaglandin E2 is generally required for human dendritic cell migration and exerts its effect via EP2 and EP4 receptors. J. Immunol. 176, 966-973 (2006).

52. Xie, J. et al. Critical roles of Raf/MEK/ERK and PI3K/AKT signaling and inactivation of p38 MAP kinase in the differentiation and survival of monocytederived immature dendritic cells. Exp. Hematol. 33, 564-572 (2005).

53. Keller, A. M., Schildknecht, A., Xiao, Y., van den Broek, M. \& Borst, J. Expression of costimulatory ligand CD70 on steady-state dendritic cells breaks CD8 + T cell tolerance and permits effective immunity. Immunity 29, 934-946 (2008).

\section{Acknowledgements}

This work was supported by startup fund from Cleveland Clinic and grants from the National Cancer Institute (R01 CA138402, R01 CA138398, R01 CA163881 and P50 CA142509), the Leukemia and Lymphoma Society and Multiple Myeloma Research Foundation.

\section{Author contributions}

Y.L. and Q.Y. initiated the study, designed the experiments and wrote the paper; Y.L. performed the majority of experiments and statistical analyses; M.Z., B.H., S.W. and. H.L. helped with ChIP and luciferase assay; Y.Z., J.Q., J.H and J.Y. provided critical suggestions to this study; and Q.Y. and J.H. contributed to the conceptual idea and to the writing and editing of the paper.

\section{Additional information}

Accession codes: The microarray data were deposited in the NCBI Gene Expression Omnibus (GEO) database under accession numbers GSE57879.

Supplementary Information accompanies this paper at http://www.nature.com/ naturecommunications

Competing financial interests: The authors declare no competing financial interests.

Reprints and permission information is available online at http://npg.nature.com/ reprintsandpermissions/

How to cite this article: Lu, Y. et al. p38 MAPK-inhibited dendritic cells induce superior antitumour immune responses and overcome regulatory T-cell-mediated immunosuppression. Nat. Commun. 5:4229 doi: 10.1038/ncomms5229 (2014). 